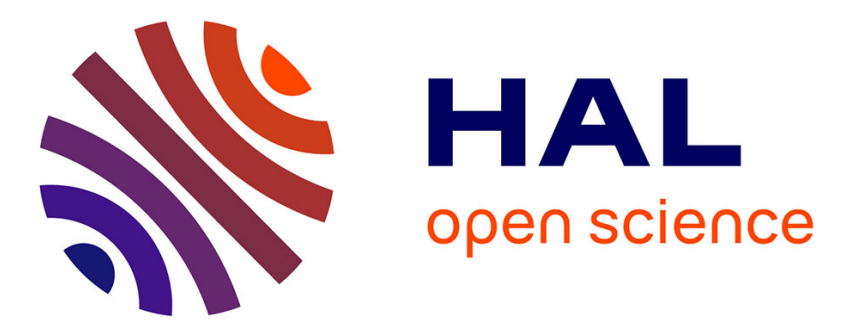

\title{
Cationic PCP and PCN NHC Core Pincer-Type Mn(I) Complexes: From Synthesis to Catalysis
}

\author{
Ruqaya Buhaibeh, Carine Guyard-Duhayon, Dmitry A. Valyaev, \\ Jean-Baptiste Sortais, Yves Canac
}

\section{- To cite this version:}

Ruqaya Buhaibeh, Carine Guyard-Duhayon, Dmitry A. Valyaev, Jean-Baptiste Sortais, Yves Canac. Cationic PCP and PCN NHC Core Pincer-Type Mn(I) Complexes: From Synthesis to Catalysis. Organometallics, 2021, 40 (2), pp.231-241. hal-03128901

\section{HAL Id: hal-03128901 https://hal.science/hal-03128901}

Submitted on 2 Feb 2021

HAL is a multi-disciplinary open access archive for the deposit and dissemination of scientific research documents, whether they are published or not. The documents may come from teaching and research institutions in France or abroad, or from public or private research centers.
L'archive ouverte pluridisciplinaire HAL, est destinée au dépôt et à la diffusion de documents scientifiques de niveau recherche, publiés ou non, émanant des établissements d'enseignement et de recherche français ou étrangers, des laboratoires publics ou privés. 


\title{
Cationic PCP and PCN NHC Core Pincer-Type Mn(I) Com- plexes: From Synthesis to Catalysis
}

\author{
Ruqaya Buhaibeh, ${ }^{\dagger}$ Carine Duhayon, ${ }^{\dagger}$ Dmitry A. Valyaev, ${ }^{\dagger, *}$ Jean-Baptiste Sortais ${ }^{\dagger, \hbar * *}$ and Yves Canac $^{\dagger, *}$ \\ † LCC-CNRS, Université de Toulouse, CNRS, UPS, 205 route de Narbonne, 31077 Toulouse Cedex 4, France. \\ $\$$ Institut Universitaire de France, 1 rue Descartes, 75231 Paris Cedex 5, France.
}

\begin{abstract}
The coordination chemistry of NHC core pincer-type PCP and PCN ligands with Mn(I) center was systematically investigated. The reaction of $\left[\mathrm{Mn}(\mathrm{CO})_{5} \mathrm{Br}\right]$ with free carbenes, conveniently prepared from the corresponding imidazolium salts and a strong base, lead to the initial formation of bidentate $f a c-\left[\mathrm{MnBr}(\mathrm{CO})_{3}\left(\kappa^{2} P, C, L\right)\right]$ complexes bearing a pending phosphine or pyridine donor extremity $(L)$, which upon bromide abstraction, afford cationic $\left[\mathrm{Mn}(\mathrm{CO})_{3}\left(\kappa^{3} P, C, L\right)\right](\mathrm{OTf})$ derivatives exhibiting a meridional or a facial coordinating geometry of tridentate NHC core ligand depending on its relative flexibility. All bi- and tridentate Mn(I) complexes were characterized by X-ray diffraction analysis. The impact of the tridentate ligand structure on catalytic performance was illustrated in the Mn-catalyzed hydrogenation of acetophenone revealing $f a c-\left[\mathrm{Mn}(\mathrm{CO})_{3}\left(\kappa^{3} P, C, N\right)\right](\mathrm{OTf})$ based on a 5,6-membered metallacyclic architecture to be the most active, thus evidencing the role of the pyridine arm lability in the catalytic cycle.
\end{abstract}

\section{INTRODUCTION}

Following the early generation of phosphines, N-heterocyclic carbenes (NHCs) have attracted much attention, leading to remarkable advances in different areas, such as organometallic chemistry, functional materials, and homogeneous catalysis. ${ }^{1}$ For catalytic purposes, it is today generally accepted that NHCs are not just phosphine mimics, but also surpass their phosphine congeners in terms of activity and application scope. ${ }^{2}$ Based on this knowledge, considerable efforts have been provided to introduce structural diversity, and a large variety of polydentate NHC ligands ${ }^{3}$ bearing coordinating atoms of different nature $(\mathrm{N}, \mathrm{P}, \mathrm{O}, \mathrm{C})$ have been prepared improving the efficiency and selectivity of metal complexation. In particular, NHC-based ligands exhibiting L-type coordinating extremities such as pyridine $^{4}$ and phosphine ${ }^{5}$ donors, or X-type such as phenolate, ${ }^{6}$ and phosphonium ylide ${ }^{7}$ donors were notably devised in the bi- and in the tridentate series extending naturally the scope of accessible metal fragments.

For catalytic applications, ${ }^{8}$ especially in the field of hydrogenation, ${ }^{9} \mathrm{Mn}$-based complexes have recently been the subject of intensive research, and many platforms have been developed over last five years. Pincer-type $\mathrm{Mn}(\mathrm{I})$ complexes based on a $\mathrm{PNP},{ }^{10} \mathrm{PNN},{ }^{11} \mathrm{NNN},{ }^{12}$ or $\mathrm{CNP}^{13}$ skeleton were reported as valuable catalysts for hydrogenation-type reactions. However, less sophisticated complexes featuring bidentate $\mathrm{PN},{ }^{14} \mathrm{PP},{ }^{15} \mathrm{NN},{ }^{16}$ $\mathrm{CN},{ }^{17}$ or $\mathrm{CP}^{18}$ scaffolds were also found to efficiently catalyze such transformations. In this competitive area of catalysis, NHC-based ligands have also demonstrated their high potential as illustrated very recently in the tridentate series for the cationic $\left[(\mathrm{CNP}) \mathrm{Mn}(\mathrm{CO})_{3}\right]^{+}$complex exhibiting a NHC-aminephosphine ligand, ${ }^{13}$ and in the bidentate series for the neutral $\left[(\mathrm{CN}) \mathrm{Mn}(\mathrm{CO})_{3} \mathrm{Br}\right]$ and $\left[(\mathrm{CP}) \mathrm{Mn}(\mathrm{CO})_{3} \mathrm{Br}\right]$ complexes based on NHC-amine ${ }^{17}$ and NHC-phosphine ${ }^{18}$ ligands, respectively.
Based on the general structure of the bidentate NHCphosphine ligand $\mathbf{A}$ recently described in our group, we wondered about the possible impact on catalytic activity of changing the 'spectator' $N$-mesityl substituent by a donor group capable of interacting with the metal center. In this contribution, we turned out attention to the preparation, the coordinating properties and the catalytic activity of the novel NHC core tridentate ligands of type $\mathbf{B}$ bearing phosphine and pyridine donor arms (Scheme 1). In a larger context, this comparative study also aimed to assess whether the improvement in catalytic performance necessarily involved the design of more complex architectures, as are pincer systems with respect to their bidentate versions.

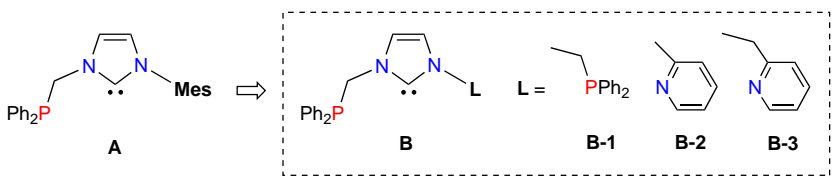

Scheme 1. Representation of recently reported bidentate NHC-phosphine ligand $A,{ }^{18}$ and related tridentate NHCcore systems B targeted in this study.

\section{RESULTS AND DISCUSSION}

Synthesis and NMR characterization of NHC-based preligands. The first representative ligand B-1 of the family was prepared from the $N$-phosphine oxide substituted imidazole 2 isolated in $70 \%$ yield by treating $1 \mathrm{H}$-imidazole 1 with 1.0 equiv. of $\mathrm{Ph}_{2} \mathrm{P}(\mathrm{O}) \mathrm{CH}_{2} \mathrm{OTs}$ in the presence of $\mathrm{K}_{2} \mathrm{CO}_{3}$ in DMSO at 90 ${ }^{\circ} \mathrm{C}$ (Scheme 2, left). The second phosphine oxide arm was then introduced by the alkylation of imidazole 2 with the same tosylate precursor in toluene at $130^{\circ} \mathrm{C}$, allowing the bis $(N$-phosphine oxide)imidazolium salt [3](OTs) to be isolated in $87 \%$ yield. The reduction of the latter under classic conditions (excess of $\mathrm{HSiCl}_{3}$ in chlorobenzene at $\left.80^{\circ} \mathrm{C}\right)^{5 \mathrm{~b}, 18}$ led to the target bis $(N$ - 


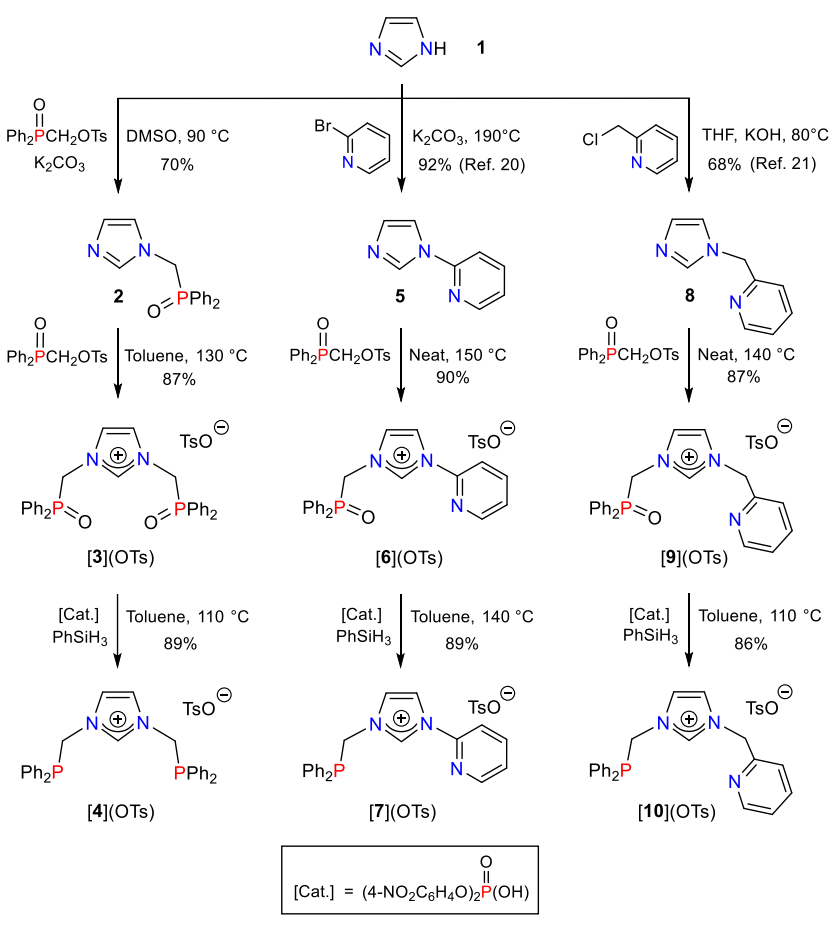

Scheme 2. Preparation of tridentate PCP and PCN pre-ligands [4](OTs), [7](OTs) and [10](OTs).

phosphino)imidazolium salt [4](OTs) in $c a .80 \%$ yield. The formation yield of this product can be improved to $89 \%$ by organocatalytic reduction approach ${ }^{19}$ by heating [3](OTs) in toluene at $110^{\circ} \mathrm{C}$ with an excess of $\mathrm{PhSiH}_{3}$ in the presence of bis(4nitrophenyl)phosphate.

The PCN ligands B-2 (Scheme 2, middle) and B-3 (Scheme 2, right) were prepared from readily available $N$-pyridyl- $\mathbf{5}^{20}$ and $N$-pyridylmethyl $\mathbf{8}^{21}$ substituted imidazoles following the same two-step strategy. In the first step, the imidazolium salts [6](OTs) and [9](OTs) featuring phosphine oxide arms were prepared in 90 and $87 \%$ yield, respectively upon solvent-free quaternization of the corresponding imidazoles with $\mathrm{Ph}_{2} \mathrm{P}(\mathrm{O}) \mathrm{CH}_{2} \mathrm{OTs}$. In both cases, the transformation of phosphine oxide moieties into phosphines using strongly reducing agent $\mathrm{HSiCl}_{3}$ was found to be non-selective, resulting in partial reduction of the pyridine nucleus. Gratifyingly, the application of the alternative organocatalytic protocol ${ }^{19}$ enabled the formation of the target pre-ligands [7](OTs) and [10](OTs) isolated in 89 and $86 \%$ yield, respectively.

The identity of all pre-ligands was mainly established on the basis of their characteristic ${ }^{31} \mathrm{P},{ }^{1} \mathrm{H}$ and ${ }^{13} \mathrm{C}$ NMR resonances (Table 1). For imidazolium salts [3](OTs), [6](OTs), and [9](OTs) exhibiting a phosphine oxide moiety, the ${ }^{31} \mathrm{P}$ NMR spectrum displayed a single signal at $\delta_{\mathrm{P}} 26.5-27.2 \mathrm{ppm}$ in the typical range for such $\mathrm{P}(\mathrm{V})$-based derivatives. As expected, the ${ }^{31} \mathrm{P}$ NMR singlet of $N$-(phosphino)imidazolium salts [4](OTs), [7](OTs), and [10](OTs) appeared at higher field at $\delta_{\mathrm{P}}-11.1$ to $-12.4 \mathrm{ppm}$ in agreement with the presence of $\mathrm{P}(\mathrm{III})$ donor extremities of alkyl(diphenyl)phosphine nature. The cationic character of these salts was confirmed by ${ }^{1} \mathrm{H}$ and ${ }^{13} \mathrm{C}$ NMR spectroscopy by the characteristic low field signals of the $\mathrm{CH}$ imidazolium fragment at $\delta_{\mathrm{H}} 9.56-10.59 \mathrm{ppm}$ and $\delta_{\mathrm{CH}} 136.0-138.6$ ppm.
Table 1. Selected ${ }^{31} \mathrm{P},{ }^{1} \mathrm{H}$ and ${ }^{13} \mathrm{C}$ NMR chemical shifts in $\mathrm{CDCl}_{3}$ (ppm) for imidazolium salts [3](OTs), [4](OTs), [6](OTs), [7](OTs), [9](OTs) and [10](OTs).

\begin{tabular}{|c|c|c|c|c|c|c|}
\hline & {$[\mathbf{3}]^{+}$} & {$[\mathbf{4}]^{+}$} & {$[\mathbf{6}]^{+}$} & {$[\mathbf{7}]^{+}$} & {$[\mathbf{9}]^{+}$} & {$[\mathbf{1 0}]^{+}$} \\
\hline$\delta_{\mathrm{P}}$ & 26.5 & -12.4 & 27.1 & -11.1 & 27.2 & -12.2 \\
\hline$\delta_{\mathrm{CH}}$ & 9.85 & 9.62 & 10.44 & 10.59 & 9.95 & 9.56 \\
\hline$\delta_{\mathrm{CH}}$ & 138.6 & 138.1 & 135.6 & 136.0 & 138.5 & 137.5 \\
\hline
\end{tabular}

Synthesis and spectroscopic characterization of NHCbased $\mathrm{Mn}(\mathrm{I})$ complexes. The complexation of pre-ligands [4](OTs), [7](OTs), and [10](OTs) was envisioned following a strategy developed recently in the case of the $N$-mesityl substituted NHC-phosphine A (Scheme 1). ${ }^{18}$ Indeed, complexes 11, 13-14, and 16 were thus readily obtained from the corresponding precursors through the sequential addition of equimolar quantities of KHMDS and [ $\left.\mathrm{Mn}(\mathrm{CO})_{5} \mathrm{Br}\right]$ in 71,80 , and $60 \%$ isolated yield, respectively (Scheme 3).
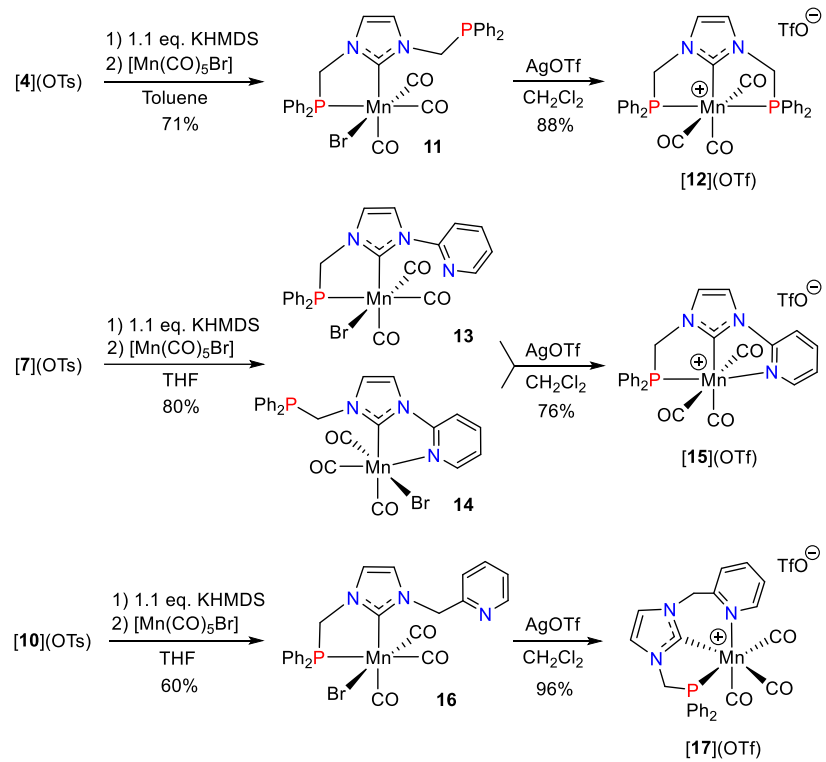

Scheme 3. Syntheses of cationic NHC-core tridentate Mn(I) complexes [12](OTf), [15](OTf), and [17](OTf) from the corresponding PCP [4](OTs) and PCN ligand precursors [7](OTs) and [10](OTs).

While only bidentate coordination was observed in all formed neutral $\mathrm{Mn}(\mathrm{I})$ complexes, a notable difference in the coordination mode was observed between the two ligands B-2 and B-3 bearing a pyridine extremity. From [7](OTs), in which the pyridine is directly connected to the imidazole core, two isomeric complexes $\mathbf{1 3}$ and $\mathbf{1 4}$ in a $c a$. 1:3 ratio were evidenced due to the possible formation of two thermodynamically favored five-membered metallacycles upon coordination of the phosphine or the pyridine arms. By contrast in the case of $[\mathbf{1 0}]$ (OTs), only the bidentate complex 16 resulting from the concomitant coordination of $\mathrm{NHC}$ and $\mathrm{PPh}_{2}$ donor units was observed, the coordination of pyridine would indeed have led to a less stable six-membered metallacycle.

With the aim of synthetizing new NHC core tridentate $\mathrm{Mn}(\mathrm{I})$ systems, the access to both neutral $\left[\left(\mathrm{L}_{3}\right) \mathrm{Mn}(\mathrm{CO})_{2} \mathrm{Br}\right]$ and cationic $\left[\left(\mathrm{L}_{3}\right) \mathrm{Mn}(\mathrm{CO})_{3}\right]^{+} \mathrm{Br}^{-}$complexes was then considered. 
Table 2. Selected ${ }^{31} \mathrm{P}$ and ${ }^{13} \mathrm{C}$ NMR chemical shifts in $\mathrm{CD}_{2} \mathrm{Cl}_{2}(\mathrm{ppm})$ with signal multiplicity and $J_{\mathrm{CP}}$ coupling constants $(\mathrm{Hz})$ in parenthesis, and IR vco frequencies in THF solution $\left(\mathrm{cm}^{-1}\right)$ with relative intensity in parenthesis for NHC-based Mn(I) complexes of bidentate $(11,13,14,16)$ and tridentate $([12]($ OTf $),[15]($ OTf $),[17]($ OTf $))$ nature.

\begin{tabular}{|c|c|c|c|c|c|c|c|}
\hline & $\mathbf{1 1}^{\mathrm{a}}$ & {$[\mathbf{1 2}](\mathrm{OTf})$} & $\mathbf{1 3}$ & $\mathbf{1 4}$ & {$[\mathbf{1 5}](\mathrm{OTf})^{\mathrm{b}}$} & $\mathbf{1 6}$ & {$[\mathbf{1 7}](\mathrm{OTf})$} \\
\hline \multirow{2}{*}{$\delta \mathrm{P}$} & $\begin{array}{c}71.0(\mathrm{~s}) \\
-17.0(\mathrm{~s})\end{array}$ & $89.6(\mathrm{~s})$ & $71.8(\mathrm{~s})$ & $-15.9(\mathrm{~s})$ & $97.4(\mathrm{~s})$ & $71.2(\mathrm{~s})$ & $93.3(\mathrm{~s})$ \\
\hline$\delta_{\mathrm{N}_{2} \mathrm{C}}$ & $197.1(\mathrm{~d}, 16.5)$ & $195.6(\mathrm{t}, 13.1)$ & $198.1(\mathrm{~d}, 15.1)$ & $206.0(\mathrm{~s})$ & $206.5(\mathrm{~d}, 18.9)$ & $197.7(\mathrm{~d}, 17.5)$ & $202.9(\mathrm{~d}, 22.3)$ \\
\hline \multirow{3}{*}{$\delta_{\mathrm{CO}}$} & $222.9(\mathrm{~d}, 17.8)$ & $220.7(\mathrm{brt}, 10.5)$, & $223.9(\mathrm{~d}, 18.2)$ & $226.4(\mathrm{~s})$ & $218.4(\mathrm{~d}, 9.7)$ & $222.8(\mathrm{brd}, 16.8)$ & $219.6(\mathrm{brd}, 25.5)$ \\
& $221.4(\mathrm{~d}, 31.0)$ & $213.6(\mathrm{brt}, 19.0)$ & $218.5(\mathrm{~d}, 32.3)$ & $218.0(\mathrm{~s})$ & $212.7(\mathrm{~d}, 17.7)$ & $220.2(\mathrm{brd}, 30.1)$ & $218.7(\mathrm{brd}, 18.5)$ \\
& $220.1(\mathrm{~d}, 21.5)$ & & $20.8)$ & $216.9(\mathrm{brd}, 23.5)$ \\
\hline \multirow{3}{*}{$v_{\mathrm{CO}}$} & $2015(\mathrm{~s})$ & $2045(\mathrm{w})$ & $2016(\mathrm{~s})$ & $2016(\mathrm{~s})$ & $2052(\mathrm{w})$ & $2018(\mathrm{~s})$ & $2024(\mathrm{~s})$ \\
& $1940(\mathrm{~s})$ & $1966(\mathrm{vs})$ & $1939(\mathrm{~s})$ & $1930(\mathrm{~s})$ & $1969(\mathrm{vs})$ & $1941(\mathrm{~s})$ & $1947(\mathrm{~s})$ \\
& $1910(\mathrm{~s})$ & $1942(\mathrm{~s})$ & $1904(\mathrm{~s})$ & $1907(\mathrm{~s})$ & $1941(\mathrm{~s})$ & $1913(\mathrm{~s})$ & $1929(\mathrm{~s})$ \\
\hline
\end{tabular}

a - IR v $v_{C O}$ frequencies were measured in a toluene solution; b - NMR data was recorded in $\mathrm{CDCl}_{3}$.

Facing the non-selective formation of $\left[\left(\mathrm{L}_{3}\right) \mathrm{Mn}(\mathrm{CO})_{2} \mathrm{Br}\right] \mathrm{com}-$ plexes upon $\mathrm{CO}$ dissociation under photochemical and thermal activation of stable bidentate $\left[\left(\kappa^{2} P, C, L\right) \mathrm{Mn}(\mathrm{CO})_{3} \mathrm{Br}\right]$ species, the latter were treated by a stoichiometric amount of AgOTf in $\mathrm{CH}_{2} \mathrm{Cl}_{2}$ to induce the bromide abstraction and thus force the coordination of a pendant donor arm. Under these conditions, airstable cationic tridentate complexes [12](OTf), [15](OTf), and [17](OTf) were easily obtained as single isomers in 88,76 , and $96 \%$ yield, respectively. These compounds belong to rare examples of NHC-containing manganese pincer-type complexes $^{13,22,23}$ and constitute the first prototypes based on NHC core $\mathrm{L}_{3}$-type ligand scaffolds.

The most pertinent spectroscopic data for the $\mathrm{Mn}(\mathrm{I}) \mathrm{NHC}$ complexes are presented in Table 2. The formation of $\mathrm{Mn}(\mathrm{I})$ NHC complexes was clearly indicated by ${ }^{1} \mathrm{H}$ and ${ }^{13} \mathrm{C} \mathrm{NMR}$ spectroscopy showing the disappearance of the $\mathrm{C}-\mathrm{H}$ imidazolium signals of pre-ligands [4](OTs), [7](OTs), and [10](OTs). Regardless of their coordination mode, in the ${ }^{13} \mathrm{C}$ NMR spectra, the $\mathrm{N}_{2} \mathrm{C}-\mathrm{Mn}$ carbon atoms of $\mathrm{NHC}$ core complexes $\left(\delta_{\mathrm{N}_{2} \mathrm{C}}\right.$ 195.6-206.5 ppm) were typically found to be strongly deshielded with proper multiplicity with respect to the $\mathrm{N}_{2} \mathrm{CH}$ carbon atoms of corresponding precursors $\left(\delta_{\mathrm{CH}} 136.0-138.6\right.$ ppm). The coordination of the $N$-substituted NHC-phosphine moiety was also apparent from the downfield shift of the ${ }^{31} \mathrm{P}$ NMR resonances, ranging from $\delta_{\mathrm{P}}-(11.1-12.4) \mathrm{ppm}$ in the preligands to $\delta \mathrm{P} 71.0-71.8 \mathrm{ppm}$ in the bidentate complexes and $\delta \mathrm{P}$ 89.6-97.4 ppm in their tridentate analogues. Solution IR spectra of all bidentate NHC $\mathrm{Mn}(\mathrm{I})$ complexes exhibited three strong $v_{\mathrm{CO}}$ bands consistent with the presence of three CO ligands in a facial arrangement. The small differences observed between these frequency values seem to indicate that the three ligands of interest have similar electronic properties. In the tridentate series, complexes [12](OTf), [15](OTf) and [17](OTf) featured three $v_{\mathrm{CO}}$ bands with different relative intensity (Table 2) suggesting two distinct $\mathrm{CO}$ ligand arrangements.

In complement to these IR frequency values, ${ }^{13} \mathrm{C}$ NMR data revealed for complexes [12](OTf) and [15](OTf) the presence of two CO resonances ([12](OTf): $\delta_{\mathrm{C}} 220.7$ (brt, $J_{\mathrm{PC}}=10.5 \mathrm{~Hz}$ ), $213.6\left(\right.$ brt, $\left.J_{\mathrm{PC}}=19.0 \mathrm{~Hz}\right)$; [15] $(\mathrm{OTf}): \delta_{\mathrm{C}} 218.4\left(\mathrm{~d}, J_{\mathrm{PC}}=9.7 \mathrm{~Hz}\right)$, $\left.212.7\left(\mathrm{~d}, J_{\mathrm{PC}}=17.7 \mathrm{~Hz}\right)\right)$, and of three resonances $\left(\delta_{\mathrm{C}} 219.6\right.$ $\left.\left(\mathrm{brd}, J_{\mathrm{PC}}=25.5 \mathrm{~Hz}\right), 218.7\left(\mathrm{~d}, J_{\mathrm{PC}}=18.5 \mathrm{~Hz}\right)\right), 216.9\left(\mathrm{~d}, J_{\mathrm{PC}}=\right.$
$23.5 \mathrm{~Hz})$ ) for [17](OTf), indicating for the latter a lower molecular symmetry than for the two former complexes and suggesting that the NHC core backbone is a priori coordinated to the metal center in a meridional fashion within complexes [12](OTf) and [15](OTf), and adopts rather a tripodal coordination mode in [17](OTf).

$\mathrm{X}$-ray diffraction structural studies of bidentate and tridentate NHC-based Mn(I) complexes. Single-crystal X-ray diffraction studies allowed us to establish the solid-state structures of the imidazolium salt [4](OTs) (Figure S1 in the Supporting Information), neutral bidentate $\mathrm{Mn}(\mathrm{I})$ complexes 13,14 and 16 (Figure 1, upper line), and their cationic tridentate congeners [12](OTf), [15](OTf) and [17](OTf) (Figure 1, lower line). The most important metrical data are presented in Table 3 . In all complexes, the hexacoordinate $\mathrm{Mn}(\mathrm{I})$ atom resides in a distorted octahedral environment. As anticipated by NMR and IR spectroscopy, three carbonyl co-ligands adopt a facial arrangement in all bidentate representatives. In the tridentate series, complexes [12](OTf) and [15](OTf) can be regarded as real pincers exhibiting the meridional coordination of the NHC core ligand with two $\mathrm{CO}$ co-ligands positioned in trans with respect to each other, while in [17](OTf) the PCN backbone acts as a tripodal ligand with the three $\mathrm{CO}$ co-ligands located cis to each other. Though such $f a c$-arrangement is less common for $\left[\mathrm{Mn}(\mathrm{CO})_{3}\left(\mathrm{~L}_{3}\right)\right]^{+}$complexes, several examples based on pincertype $\mathrm{PNP},{ }^{24} \mathrm{CNP},{ }^{13} \mathrm{NNN},{ }^{12,25} \mathrm{NNS}^{26}$ and $\mathrm{NSN}^{27}$ ligands have been recently reported.

In the pincer systems, the two coordinating heteroatoms are positioned in pseudo-trans position with respect to the metal center ([12](OTf): P1-Mn1-P2 = 160.148(14); [15](OTf): $\left.\mathrm{P} 1-\mathrm{Mn} 1-\mathrm{N} 3=153.76(4)^{\circ}\right)$ as opposed to the tripodal complex [17](OTf) where the related coordination angle is much more acute $\left(\mathrm{P} 1-\mathrm{Mn} 1-\mathrm{N} 3=91.92(3)^{\circ}\right)$. As generally observed in mer- $\mathrm{Mn}(\mathrm{I})$ complexes, the $\mathrm{C}-\mathrm{O}$ distances in the mutually trans CO ligands are shorter ([12](OTf): 1.1407(17), 1.1383(17) А; [15](OTf): $1.139(2), 1.138(2) \AA)$ than in the CO trans to the ligand, in this case the NHC core ([12](OTf): 1.1423(16) $\AA$; [15](OTf): $1.151(2) \AA) .{ }^{28}$ The PCP and PCN pincer structures [12](OTf) and [15](OTf) are defined by the presence of two fused 5-membered metallacycles with the PCP backbone in the former symmetrically linked to $\mathrm{Mn}$ through two quasi-equal P-Mn bonds (2.2627(3) and 2.2661(3) Å). The facial geometry 


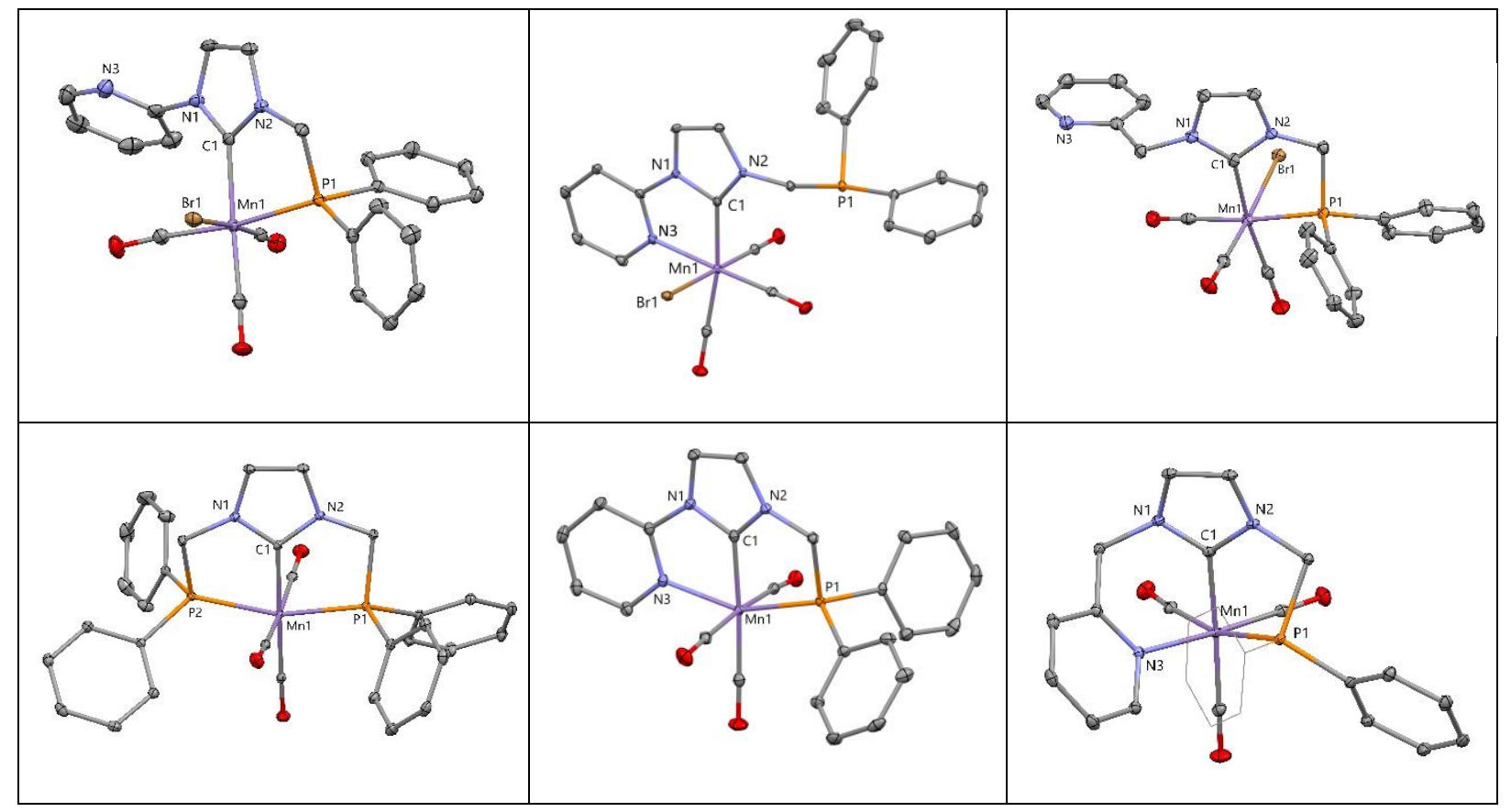

Figure 1. Perspective views of bidentate (13, 14, 16, upper line), and tridentate ([12](OTf), [15](OTf), [17](OTf), lower line) Mn(I) NHC complexes with thermal ellipsoids drawn at the $30 \%$ probability level. The hydrogen atoms are omitted for clarity.

Table 3. Selected bond lengths $(\AA)$ and bond angles $\left(^{\circ}\right)$ for bidentate and tridentate Mn(I) NHC complexes.

\begin{tabular}{|c|c|c|c|c|c|c|}
\hline Complex & $\mathbf{1 3}$ & $\mathbf{1 4}$ & $\mathbf{1 6}$ & {$[\mathbf{1 2}](\mathrm{OTf})$} & {$[\mathbf{1 5}](\mathrm{OTf})$} & {$[\mathbf{1 7}](\mathrm{OTf})$} \\
\hline $\mathrm{C} 1-\mathrm{Mn} 1$ & $2.054(2)$ & $1.993(2)$ & $2.049(3)$ & $1.9726(12)$ & $1.9360(16)$ & $1.9881(11)$ \\
\hline $\mathrm{P} 1-\mathrm{Mn} 1$ & $2.3175(7)$ & - & $2.2861(9)$ & $2.2627(3)$ & $2.2712(5)$ & $2.3348(3)$ \\
\hline $\mathrm{P} 2-\mathrm{Mn} 1$ & - & - & - & $2.2661(3)$ & - & - \\
\hline $\mathrm{N} 3-\mathrm{Mn} 1$ & - & $2.0753(17)$ & - & - & $2.0894(14)$ & $2.1095(9)$ \\
\hline $\mathrm{Mn} 1-\mathrm{Br}$ & $2.5510(5)$ & $2.5354(4)$ & $2.5527(5)$ & - & - & - \\
\hline $\mathrm{C} 1-\mathrm{Mn} 1-\mathrm{P} 1$ & $79.02(7)$ & - & $79.95(9)$ & $79.88(4)$ & $78.15(5)$ & $75.16(6)$ \\
\hline $\mathrm{C} 1-\mathrm{Mn} 1-\mathrm{P} 2$ & - & - & - & $80.30(4)$ & - & - \\
\hline $\mathrm{C} 1-\mathrm{Mn} 1-\mathrm{N} 3$ & - & $78.17(8)$ & - & - & $75.67(6)$ & $82.90(4)$ \\
\hline $\mathrm{P} 1-\mathrm{Mn} 1-\mathrm{P} 2$ & - & - & - & $160.148(14)$ & - & - \\
\hline $\mathrm{P} 1-\mathrm{Mn} 1-\mathrm{N} 3$ & - & - & - & - & $153.76(4)$ & $91.92(3)$ \\
\hline
\end{tabular}

observed in [17](OTf) can be rationalized by the presence of a longer lateral chain which offers more flexibility accommodating the formation of a six-membered metallacycle upon pyridine coordination. ${ }^{29}$ As general trend, the $\mathrm{Mn}-\mathrm{NHC}$ bond distances are significantly shortened in the tridentate systems compared to their bidentate precursors, a difference that may be attributed to the more constrained geometries and the cationic nature of the pincer-type complexes. It is noteworthy, that the Mncarbene bond in pincer complex [15](OTf) $(1.9360(16) \AA)$ is significantly shorter than that observed in binuclear $\left[\mathrm{Mn}_{2}(\mathrm{CO})_{7}(\mathrm{PCN})\right]$ complex bearing structurally close tridentate PCN ligand in the bridging $\mu-\kappa^{2} C, N-\mathrm{M} ; \kappa^{1} P$-M-coordination mode $(1.9700(17) \AA)^{30}$ and indeed represents one of the shortest distance known to date for manganese imidazol-2-ylidene complexes. ${ }^{31}$ Since a similar trend was previously observed for re- lated $\mathrm{Ni}(\mathrm{II})$ complex, ${ }^{4 \mathrm{~d}}$ it may be assumed that the rigid character of such PCN ligands forming two five-membered metallacycles is particularly favorable to strengthen metal-NHC bonding.

Catalytic investigations. Based on the recent observation that a $\mathrm{Mn}(\mathrm{I})$ complex of the bidentate NHC-phosphine ligand A behaves as one of the most efficient Mn-based catalytic system for the hydrogenation of ketones, ${ }^{18}$ this transformation was naturally selected to evaluate the influence of an additional coordinating arm. For such comparative study, three pincer-type complexes [12](OTf), [15](OTf), and [17](OTf) differing only by the nature of one donor end were considered. The catalytic tests were performed under $\mathrm{H}_{2}$ pressure (50 bar) in $t$-AmOH at 60 or $100{ }^{\circ} \mathrm{C}$, and in the presence of $10 \mathrm{~mol} \%$ of $t$-BuOK (Table 4). Gratifyingly at $100{ }^{\circ} \mathrm{C}$, in the presence of $1.0 \mathrm{~mol} \%$ of [17](OTf), acetophenone was fully reduced to 1-phenylethanol (entry 9). The catalytic charge could be decreased to $0.5 \mathrm{~mol} \%$ 
at the same temperature keeping a good conversion of $80 \%$ (entry 10 ). At $60^{\circ} \mathrm{C}$, the alcohol was produced in lower yields with a conversion of 30 and $20 \%$ by using 1.0 and $0.5 \mathrm{~mol} \%$ of [17](OTf), respectively (entries 11-12). Noteworthy, no reaction took place in the absence of base. The two other selected complexes were found to be significantly less active in this process. For instance, at $100{ }^{\circ} \mathrm{C}$, with a catalytic charge of 1.0 mol\% of pre-catalysts [12](OTf) and [15](OTf), the acetophenone was only converted into the corresponding alcohol in 27 and $39 \%$ yields, respectively (entries 1 and 5). The superiority of fac-complex [17](OTf) was tentatively attributed to the presence of a six-membered metallacycle resulting in greater lability of the pyridine arm and thus allowing the release of a vacant site during the catalytic cycle. ${ }^{13}$ The lability of a donor extremity was also supported in other systems by the catalytic activities of the bidentate complexes 11, 14 and 16 which were found to be very close to those obtained with their corresponding tridentate forms [12](OTf), [15](OTf), and [17](OTf) (entries 13$15)$. These results suggest that the same type of catalytic active species is certainly involved in both series. Noteworthy, the performance of complex [17](OTf) remains lower than that of the parent complex based on the bidentate NHC-phosphine ligand $\mathbf{A},{ }^{18}$ showing that the development of more elaborate structures is not always a prerequisite to reach higher activities in the field of catalysis.

Table 4. Catalytic hydrogenation of acetophenone with NHC core $M n(I)$ complexes [12](OTf), [15](OTf), and $[17](\mathbf{O T f}) .^{\mathrm{a}}$

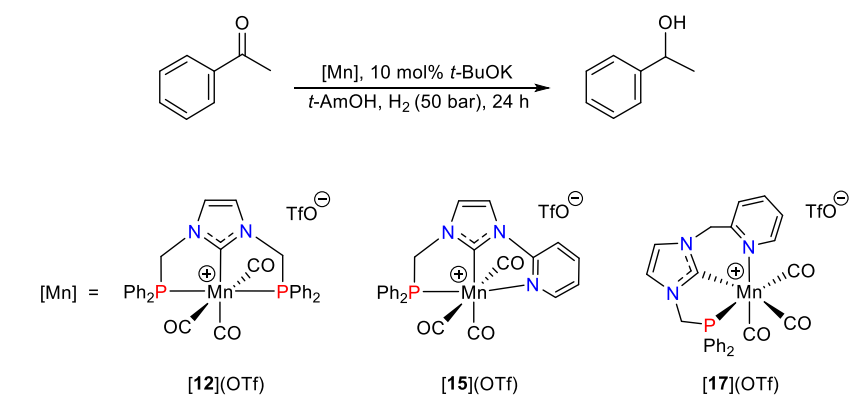

\begin{tabular}{|c|c|c|c|c|}
\hline Entry & Complex & Cat. $(\mathrm{mol} \%)$ & Temp. $\left({ }^{\circ} \mathrm{C}\right)$ & Conv. $\%^{\mathrm{b}}$ \\
\hline 1 & {$[\mathbf{1 2}](\mathrm{OTf})$} & 1.0 & 100 & 27 \\
\hline 2 & {$[\mathbf{1 2}](\mathrm{OTf})$} & 0.5 & 100 & 19 \\
\hline 3 & {$[\mathbf{1 2}](\mathrm{OTf})$} & 1.0 & 60 & 6 \\
\hline 4 & {$[\mathbf{1 2}](\mathrm{OTf})$} & 0.5 & 60 & 3 \\
\hline 5 & {$[\mathbf{1 5}](\mathrm{OTf})$} & 1.0 & 100 & 39 \\
\hline 6 & {$[\mathbf{1 5}](\mathrm{OTf})$} & 0.5 & 100 & 21 \\
\hline 7 & {$[\mathbf{1 5}](\mathrm{OTf})$} & 1.0 & 60 & 22 \\
\hline 8 & {$[\mathbf{1 5}](\mathrm{OTf})$} & 0.5 & 60 & 11 \\
\hline 9 & {$[\mathbf{1 7}](\mathrm{OTf})$} & 1.0 & 100 & $100(95)$ \\
\hline 10 & {$[\mathbf{1 7}](\mathrm{OTf})$} & 0.5 & 100 & 80 \\
\hline 11 & {$[\mathbf{1 7}](\mathrm{OTf})$} & 1.0 & 60 & 30 \\
\hline 12 & {$[\mathbf{1 7}](\mathrm{OTf})$} & 0.5 & 60 & 20 \\
\hline 13 & $\mathbf{1 1}$ & 1.0 & 100 & 42 \\
\hline 14 & $\mathbf{1 4}$ & 1.0 & 100 & 33 \\
\hline
\end{tabular}

\begin{tabular}{|l|l|l|l|l|}
\hline 15 & $\mathbf{1 6}$ & 1.0 & 100 & 98 \\
\hline
\end{tabular}

a - Procedure: an autoclave was charged with a pre-catalyst, acetophenone (1.0 mmol), $t$-BuOK $(10 \mathrm{~mol} \%)$ in $t$-AmOH $(2 \mathrm{~mL})$ under $\mathrm{H}_{2}\left(50\right.$ bar) at 60 or $100{ }^{\circ} \mathrm{C}$. b - Conversions determined by GC and ${ }^{1} \mathrm{H} \mathrm{NMR}$, isolated yield in parenthesis.

\section{CONCLUSION}

A family of cationic NHC-phosphine $\mathrm{Mn}(\mathrm{I})$ pincer-type complexes $\left[\mathrm{Mn}(\mathrm{CO})_{3}\left(\kappa^{3} P, C, L\right]\right)(\mathrm{OTf})$ with a variable third donor extremity were efficiently prepared from the readily available imidazolium salts by a sequence of free NHCs coordination to $\left[\mathrm{Mn}(\mathrm{CO})_{5} \mathrm{Br}\right]$ and bromide abstraction in the resulting bidentate complexes $f a c-\left[\mathrm{MnBr}(\mathrm{CO})_{3}\left(\kappa^{2} P, C, L\right)\right]$. The complete series of bi- and tridentate $\mathrm{Mn}(\mathrm{I})$ species have been fully characterized and systematically studied by X-ray diffraction analysis revealing in particular two different coordination modes for the nonsymmetrical PCN ligands in complexes mer$\left[\mathrm{Mn}(\mathrm{CO})_{3}\left(\kappa^{3} P, C, N\right)\right](\mathrm{OTf}) \quad([\mathbf{1 5}](\mathrm{OTf}))$ and $\mathrm{fac}$ $\left[\mathrm{Mn}(\mathrm{CO})_{3}\left(\kappa^{3} P, C, N\right)\right](\mathrm{OTf})([\mathbf{1 7}](\mathrm{OTf}))$ rationalized by the higher flexibility of ligand with $\mathrm{CH}_{2} \mathrm{Py}$ extremity in the latter case. The evaluation of these compounds in catalytic hydrogenation of acetophenone revealed the fac$\left[\mathrm{Mn}(\mathrm{CO})_{3}\left(\kappa^{3} P, C, N\right)(\mathrm{OTf})\right.$ pre-catalyst to be the most active system, the efficiency of which was attributed to the presence of a 5,6-fused membered architecture promoting the dissociation of the pyridine arm and thereby releasing a vacant site beneficial to the catalytic process. Future studies will aim to engage these $\mathrm{Mn}(\mathrm{I})$ complexes in other catalytic processes, but also to implant this new class of NHC-core pincer-type ligands on different transition metal centers.

\section{EXPERIMENTAL SECTION}

General Remarks. All manipulations were performed under an inert atmosphere of dry nitrogen by using standard vacuum line and Schlenk tube techniques. Glassware was dried at $120^{\circ} \mathrm{C}$ in an oven for at least three hours. Dry and oxygen-free organic solvents (THF, $\mathrm{Et}_{2} \mathrm{O}$, $\mathrm{CH}_{2} \mathrm{Cl}_{2}$, toluene, pentane) were obtained using a LabSolv (Innovative Technology) solvent purification system. Acetonitrile was dried and distilled over $\mathrm{P}_{2} \mathrm{O}_{5}$ under argon. Chromatographic purification was carried out on silica gel $\left(\mathrm{SiO}_{2}, 63-200 \mu \mathrm{m}\right)$ and activated basic alumina (Brockmann I). ${ }^{1} \mathrm{H},{ }^{31} \mathrm{P}$, and ${ }^{13} \mathrm{C}$ NMR spectra were obtained on Bruker AV300, AV400 or NEO600 spectrometers. NMR chemical shifts $\delta$ are in ppm, with positive values to high frequency relative to the tetramethylsilane reference for ${ }^{1} \mathrm{H}$ and ${ }^{13} \mathrm{C}$ and to $85 \% \mathrm{H}_{3} \mathrm{PO}_{4}$ for ${ }^{31} \mathrm{P}$. If necessary, additional information on the carbon signal attribution was obtained using ${ }^{13} \mathrm{C}\left\{{ }^{1} \mathrm{H},{ }^{31} \mathrm{P}\right\}, J$-modulated spin-echo (JMOD) ${ }^{13} \mathrm{C}\left\{{ }^{1} \mathrm{H}\right\}$, ${ }^{1} \mathrm{H}-{ }^{13} \mathrm{C}$ HMQC, and/or HMBC experiments. Solution IR spectra were recorded in $0.1 \mathrm{~mm} \mathrm{CaF}_{2}$ cells using a Perkin Elmer Frontier FT-IR spectrometer and given in $\mathrm{cm}^{-1}$ with relative intensity in parentheses. GC analyses were performed with GC-2014 (Shimadzu) 2010 equipped with a capillary column (Supelco, SPBTM-20, fused silica capillary column, $30 \mathrm{~m} \times 0.25 \mathrm{~mm} \times 0.25 \mathrm{~mm}$ film thickness). Highresolution mass spectra (ESI positive mode) were obtained using a Xevo G2 QTof (Waters) spectrometer. Elemental analyses were carried out at the LCC-CNRS (Toulouse) using a Perkin Elmer 2400 series II analyzer. Non-stirred 'Autoclave Maxitech' autoclaves $(50 \mathrm{~mL})$ were used for the hydrogenation. $\mathrm{Ph}_{2} \mathrm{P}(\mathrm{O}) \mathrm{CH}_{2} \mathrm{OTs},{ }^{18}$ 2-(1H-imidazol-1yl)pyridine $\mathbf{5},{ }^{20}$ and 2-(1H-imidazol-1-yl-methyl)pyridine $\mathbf{8}^{21}$ were prepared according to known methods. All other reagent grade chemicals purchased from commercial sources were used as received.

Synthesis of $\left[\mathrm{Ph}_{2} \mathbf{P}(\mathbf{O}) \mathbf{C H}_{2} \mathbf{I m}\right]$ (2). A Schlenk tube was charged with $\mathrm{Ph}_{2} \mathrm{P}(\mathrm{O}) \mathrm{CH}_{2} \mathrm{OTs}(4.0 \mathrm{~g}, 10.4 \mathrm{mmol}), 1 \mathrm{H}$-imidazole $(0.71 \mathrm{~g}, 10.4$ mmol) and $\mathrm{K}_{2} \mathrm{CO}_{3}(9.67 \mathrm{~g}, 70.0 \mathrm{mmol})$ under an air atmosphere. After addition of DMSO $(40 \mathrm{~mL})$, the suspension was vigorously stirred at $90{ }^{\circ} \mathrm{C}$ for $24 \mathrm{~h}$. The reaction mixture was cooled to $50{ }^{\circ} \mathrm{C}$ and DMSO was removed under reduced pressure. The solid residue was dissolved 
in $\mathrm{CHCl}_{3}(20 \mathrm{~mL})$, the solution was washed several times with $\mathrm{H}_{2} \mathrm{O}$, dried over $\mathrm{MgSO}_{4}$ and evaporated under reduced pressure. The resulting solid was washed with $\mathrm{Et}_{2} \mathrm{O}(20 \mathrm{~mL})$ and dried under vacuum to afford 2 as a white powder $(1.98 \mathrm{~g}, 70 \%) .{ }^{1} \mathrm{H}$ NMR $\left(400 \mathrm{MHz}, \mathrm{CDCl}_{3}\right.$, $\left.25{ }^{\circ} \mathrm{C}\right): \delta 7.70-7.65\left(\mathrm{~m}, 4 \mathrm{H}, \mathrm{C} H_{\mathrm{Ph}}\right), 7.61-7.57\left(\mathrm{~m}, 2 \mathrm{H}, \mathrm{C} H_{\mathrm{Ph}}\right), 7.52-$ 7.47 (m, 4H, CHPh), 7.30 (brs, $1 \mathrm{H}, \mathrm{CH}_{\mathrm{Im}-2}$ ), 6.96 (brs, $1 \mathrm{H}, \mathrm{C} H_{\mathrm{Im}-4,5}$ ), 6.91 (brs, $1 \mathrm{H}, \mathrm{CH}_{\mathrm{Im}-4,5)}, 4.77\left(\mathrm{~d}, J_{\mathrm{PH}}=4.5 \mathrm{~Hz}, 2 \mathrm{H}, \mathrm{CH}_{2}\right) ;{ }^{31} \mathrm{P}\left\{{ }^{1} \mathrm{H}\right\} \mathrm{NMR}$ $\left(162 \mathrm{MHz}, \mathrm{CDCl}_{3}, 25{ }^{\circ} \mathrm{C}\right): \delta 25.7(\mathrm{~s}) ;{ }^{13} \mathrm{C}\left\{{ }^{1} \mathrm{H}\right\} \mathrm{NMR}\left(101 \mathrm{MHz}, \mathrm{CDCl}_{3}\right.$, $\left.25^{\circ} \mathrm{C}\right): \delta 137.9\left(\mathrm{brs}, C \mathrm{H}_{\mathrm{Im}-2}\right), 132.9\left(\mathrm{~d}, J_{\mathrm{PC}}=2.5 \mathrm{~Hz}, C \mathrm{H}_{\mathrm{Ph}}\right), 131.2(\mathrm{~d}$,

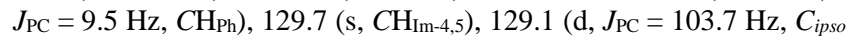
Ph), $129.0\left(\mathrm{~d}, J_{\mathrm{PC}}=18.0 \mathrm{~Hz}, C \mathrm{H}_{\mathrm{Ph}}\right), 120.3\left(\mathrm{~s}, C \mathrm{H}_{\mathrm{Im}-4.5}\right), 47.8\left(\mathrm{~d}, J_{\mathrm{PC}}=\right.$ $73.9 \mathrm{~Hz}, \mathrm{CH}_{2}$ ); Anal. Found: C, 65.58; H, 5.63; N, 9.48. Calcd. (\%) for $\mathrm{C}_{16} \mathrm{H}_{15} \mathrm{~N}_{2} \mathrm{OP}$. 0.25 (DMSO): C, 65.66; H, 5.51; N, 9.28; MS (ES $\left.{ }^{+}\right): m / z$ : $283.1[\mathrm{M}+\mathrm{H}]^{+}$; HRMS $\left(\mathrm{ES}^{+}\right)$: calcd. for $\mathrm{C}_{16} \mathrm{H}_{16} \mathrm{~N}_{2} \mathrm{OP}^{+} 283.1000$; found, $283.1006\left(\varepsilon_{\mathrm{r}}=2.1 \mathrm{ppm}\right)$.

Synthesis of $\left[\left(\mathrm{Ph}_{2} \mathrm{P}(\mathrm{O}) \mathrm{CH}_{2}\right)_{2} \mathrm{Im}\right] \mathrm{OTs}$ [3](OTs). A mixture of 2 (1.26 g, $4.5 \mathrm{mmol})$ and $\mathrm{Ph}_{2} \mathrm{P}(\mathrm{O}) \mathrm{CH}_{2} \mathrm{OTs}(1.70 \mathrm{~g}, 4.5 \mathrm{mmol})$ was heated under stirring in toluene $(5 \mathrm{~mL})$ at $130{ }^{\circ} \mathrm{C}$ for $16 \mathrm{~h}$. After cooling to RT, the light-brown solid formed was dissolved in $\mathrm{CH}_{2} \mathrm{Cl}_{2}(5 \mathrm{~mL})$ and then $\mathrm{Et}_{2} \mathrm{O}(50 \mathrm{~mL})$ was added. The resulting precipitate was triturated with $\mathrm{Et}_{2} \mathrm{O}(20 \mathrm{~mL})$ until the formation of a white powder $(\mathrm{ca} .30 \mathrm{~min})$. The supernatant was then removed by decantation and the precipitate was washed with $\mathrm{Et}_{2} \mathrm{O}(10 \mathrm{~mL})$ and dried under vacuum to afford [3](OTs) as a white powder $(2.6 \mathrm{~g}, 87 \%) .{ }^{1} \mathrm{H} \mathrm{NMR}\left(400 \mathrm{MHz}, \mathrm{CDCl}_{3}, 25{ }^{\circ} \mathrm{C}\right): \delta$ $9.85\left(\mathrm{~s}, 1 \mathrm{H}, \mathrm{C} H_{\mathrm{Im}-2}\right), 7.82-7.77\left(\mathrm{~m}, 10 \mathrm{H}, \mathrm{CH}_{\mathrm{Ar}}\right), 7.48-7.42(\mathrm{~m}, 6 \mathrm{H}$, $\left.\mathrm{CH}_{\mathrm{Ar}}\right), 7.34-7.30\left(\mathrm{~m}, 8 \mathrm{H}, \mathrm{C} H_{\mathrm{Ar}}\right), 7.08\left(\mathrm{~d}, J_{\mathrm{HH}}=7.8 \mathrm{~Hz}, 2 \mathrm{H}, \mathrm{C} H_{\mathrm{Ts}}\right), 5.28$ $\left(\mathrm{d}, J_{\mathrm{PH}}=6.3 \mathrm{~Hz}, 4 \mathrm{H}, \mathrm{CH}\right), 2.30\left(\mathrm{~s}, 3 \mathrm{H}, \mathrm{CH}_{3 \mathrm{Ts}}\right) ;{ }^{31} \mathrm{P}\left\{{ }^{1} \mathrm{H}\right\} \mathrm{NMR}(162$ $\left.\mathrm{MHz}, \mathrm{CDCl}_{3}, 25{ }^{\circ} \mathrm{C}\right): \delta 26.5(\mathrm{~s}) ;{ }^{13} \mathrm{C}\left\{{ }^{1} \mathrm{H}\right\}$ NMR (101 MHz, $\mathrm{CDCl}_{3}, 25$ $\left.{ }^{\circ} \mathrm{C}\right): \delta 143.6\left(\mathrm{~s}, C_{\mathrm{Ts}}\right), 139.6\left(\mathrm{~s}, C_{\mathrm{Ts}}\right), 138.6\left(\mathrm{~s}, C_{\mathrm{Im}-2}\right), 133.0\left(\mathrm{~d}, J_{\mathrm{PC}}=\right.$ $\left.2.0 \mathrm{~Hz}, C \mathrm{H}_{\mathrm{Ph}}\right), 131.3\left(\mathrm{~d}, J_{\mathrm{PC}}=9.9 \mathrm{~Hz}, C \mathrm{H}_{\mathrm{Ph}}\right), 129.2\left(\mathrm{~d}, J_{\mathrm{PC}}=12.5 \mathrm{~Hz}\right.$, $C \mathrm{HPh}_{\mathrm{Ph}}, 128.8\left(\mathrm{~s}, C \mathrm{H}_{\mathrm{Ts}}\right), 127.9$ (d, $\left.J_{\mathrm{PC}}=103.8 \mathrm{~Hz}, C_{i p s o} \mathrm{Ph}\right), 126.0(\mathrm{~s}$, $\left.C \mathrm{H}_{\mathrm{Ts}}\right), 123.2\left(\mathrm{~s}, C \mathrm{H}_{\mathrm{Im}-4,5}\right), 48.6\left(\mathrm{~d}, J_{\mathrm{PC}}=66.0 \mathrm{~Hz}, C \mathrm{H}_{2}\right), 21.4\left(\mathrm{~s}, C \mathrm{H}_{3 \mathrm{Ts}}\right)$; Anal. Found: C, 59.71; H, 4.97; N, 3.84. Calcd. (\%) for $\mathrm{C}_{36} \mathrm{H}_{34} \mathrm{~N}_{2} \mathrm{O}_{5} \mathrm{P} 2 \mathrm{~S} .0 .85\left(\mathrm{CH}_{2} \mathrm{Cl}_{2}\right): \mathrm{C}, 59.74 ; \mathrm{H}, 4.86 ; \mathrm{N}, 3.78 ; \mathrm{MS}\left(\mathrm{ES}^{+}\right)$: $m / z$ : $497.1[\mathrm{M}]^{+}$; HRMS (ES ${ }^{+}$): calcd. for $\mathrm{C}_{29} \mathrm{H}_{27} \mathrm{~N}_{2} \mathrm{O}_{2} \mathrm{P}_{2}{ }^{+} 497.1548$; found, $497.1534\left(\varepsilon_{\mathrm{r}}=2.8 \mathrm{ppm}\right)$.

Synthesis of $\left[\left(\mathrm{Ph}_{2} \mathbf{P C H}_{2}\right)_{2} \mathrm{Im}\right] \mathrm{OTs}$ [4](OTs). A Schlenk tube was charged with bis(4-nitrophenyl)phosphate $(408 \mathrm{mg}, 1.2 \mathrm{mmol})$ and [3](OTs) $(4.0 \mathrm{~g}, 6.0 \mathrm{mmol})$ under $\mathrm{N}_{2}$ flow. Dry toluene ( $\left.40 \mathrm{~mL}\right), \mathrm{PhSiH}_{3}$ $(6 \mathrm{~mL}, 48 \mathrm{mmol})$ were added and the mixture was stirred at $110{ }^{\circ} \mathrm{C}$ for $16 \mathrm{~h}$. After cooling to RT, the volatiles were removed under vacuum. The resulting solid was dissolved in degassed $\mathrm{CH}_{2} \mathrm{Cl}_{2}(10 \mathrm{~mL})$ and then degassed $\mathrm{Et}_{2} \mathrm{O}$ was added $(100 \mathrm{~mL})$. The resulting precipitate was washed with degassed $\mathrm{Et}_{2} \mathrm{O}(30 \mathrm{~mL})$ and dried under vacuum to afford [4](OTs) as a beige powder $(3.4 \mathrm{~g}, 89 \%)$. Single crystals suitable for XRD were obtained by slow evaporation of a concentrated $\mathrm{CH}_{2} \mathrm{Cl}_{2}$ solution at RT. ${ }^{1} \mathrm{H} \mathrm{NMR}\left(400 \mathrm{MHz}, \mathrm{CDCl}_{3}, 25{ }^{\circ} \mathrm{C}\right): \delta 9.62\left(\mathrm{~s}, 1 \mathrm{H}, \mathrm{CH}_{\mathrm{Im}-}\right.$ 2), $7.79\left(\mathrm{~d}, J_{\mathrm{HH}}=8.1 \mathrm{~Hz}, 2 \mathrm{H}, \mathrm{C} H_{\mathrm{Ts}}\right), 7.43-7.30\left(\mathrm{~m}, 20 \mathrm{H}, \mathrm{C} H_{\mathrm{Ph}}\right), 7.10$ $\left(\mathrm{d}, J_{\mathrm{HH}}=7.9 \mathrm{~Hz}, 2 \mathrm{H}, \mathrm{CH} H_{\mathrm{Ts}}\right), 6.88$ (brs, $2 \mathrm{H}, \mathrm{C} H_{\mathrm{Im}-4,5)}, 4.92\left(\mathrm{~d}, J_{\mathrm{PH}}=5.5\right.$ $\left.\mathrm{Hz}, 4 \mathrm{H}, \mathrm{CH}_{2}\right), 2.31$ (s, 3H, CH $\left.3 \mathrm{Ts}\right) ;{ }^{31} \mathrm{P}\left\{{ }^{1} \mathrm{H}\right\}$ NMR $\left(162 \mathrm{MHz}, \mathrm{CDCl}_{3}\right.$, $\left.25{ }^{\circ} \mathrm{C}\right): \delta-12.4(\mathrm{~s}) ;{ }^{13} \mathrm{C}\left\{{ }^{1} \mathrm{H}\right\} \mathrm{NMR}\left(101 \mathrm{MHz}, \mathrm{CDCl}_{3}, 25{ }^{\circ} \mathrm{C}\right): \delta 143.9$ $\left(\mathrm{s}, C_{\mathrm{Ts}}\right), 139.3\left(\mathrm{~s}, C_{\mathrm{Ts}}\right), 138.1\left(\mathrm{~s}, C \mathrm{H}_{\mathrm{Im}-2}\right), 133.1\left(\mathrm{~d}, J_{\mathrm{PC}}=19.8 \mathrm{~Hz}, C \mathrm{H}_{\mathrm{Ph}}\right)$, $133.0\left(\mathrm{~d}, J_{\mathrm{PC}}=13.1 \mathrm{~Hz}, C_{i p s o} \mathrm{Ph}\right), 130.2\left(\mathrm{~s}, C \mathrm{H}_{\mathrm{Ph}}\right), 129.3\left(\mathrm{~d}, J_{\mathrm{PC}}=7.0\right.$ $\left.\mathrm{Hz}, C \mathrm{H}_{\mathrm{Ph}}\right), 128.7\left(\mathrm{~s}, C \mathrm{H}_{\mathrm{Ts}}\right), 126.2\left(\mathrm{~s}, C \mathrm{H}_{\mathrm{Ts}}\right), 121.9\left(\mathrm{~d}, J_{\mathrm{PC}}=4.2 \mathrm{~Hz}, C \mathrm{H}\right.$ Im-4,5), 48.9 (d, $\left.J_{\mathrm{PC}}=21.1 \mathrm{~Hz}, C_{2}\right), 21.4\left(\mathrm{~s}, C_{3 \mathrm{Ts}}\right)$; Anal. Found: $\mathrm{C}$, 65.83; H, 5.19; N, 4.25. Calcd. (\%) for $\mathrm{C}_{36} \mathrm{H}_{34} \mathrm{~N}_{2} \mathrm{O}_{3} \mathrm{P}_{2} \mathrm{~S} .0 .30\left(\mathrm{CH}_{2} \mathrm{Cl}_{2}\right)$; C, 65.84; H, 5.27; N, 4.23; MS (ES $\left.{ }^{+}\right): m / z: 465.1\left[\mathrm{M}^{+}\right.$; HRMS $\left(\mathrm{ES}^{+}\right)$: calcd. for $\mathrm{C}_{29} \mathrm{H}_{27} \mathrm{~N}_{2} \mathrm{P}_{2}{ }^{+} 465.1649$; found, $465.1648\left(\varepsilon_{\mathrm{r}}=0.2 \mathrm{ppm}\right)$.

Synthesis of $\left[\mathrm{Ph}_{2} \mathbf{P}(\mathrm{O}) \mathrm{CH}_{2} \mathrm{ImPy}\right] \mathrm{OTs}$ [6](OTs). A mixture of 5 $(0.68 \mathrm{~g}, 4.7 \mathrm{mmol})$ and $\mathrm{Ph}_{2} \mathrm{P}(\mathrm{O}) \mathrm{CH}_{2} \mathrm{OTs}(1.5 \mathrm{~g}, 3.9 \mathrm{mmol})$ was heated under stirring at $150{ }^{\circ} \mathrm{C}$ for $16 \mathrm{~h}$. After cooling to RT, the resulting brown solid was dissolved in $\mathrm{CH}_{2} \mathrm{Cl}_{2}(5 \mathrm{~mL})$ and then $\mathrm{Et}_{2} \mathrm{O}(100 \mathrm{~mL})$ was added. The resulting precipitate was triturated with $\mathrm{Et}_{2} \mathrm{O}(30 \mathrm{~mL})$ until the formation of a white powder (ca. $30 \mathrm{~min})$. The supernatant was removed by decantation and the precipitate was washed with $\mathrm{Et}_{2} \mathrm{O}$ $(10 \mathrm{~mL})$ and dried under vacuum to afford [6](OTs) as a very hygroscopic white powder $(1.8 \mathrm{~g}, 90 \%) .{ }^{1} \mathrm{H} \mathrm{NMR}\left(400 \mathrm{MHz}, \mathrm{CDCl}_{3}, 25{ }^{\circ} \mathrm{C}\right)$ : $\delta 10.44\left(\mathrm{~s}, 1 \mathrm{H}, \mathrm{C} H_{\mathrm{Im}-2}\right), 8.34\left(\mathrm{~d}, J_{\mathrm{HH}}=4.6 \mathrm{~Hz}, 1 \mathrm{H}, \mathrm{C} H_{\mathrm{Ar}}\right), 8.08(\mathrm{~s}, 1 \mathrm{H}$, $\left.\mathrm{CH}_{\mathrm{Ar}}\right), 7.99-7.94\left(\mathrm{~m}, 5 \mathrm{H}, \mathrm{C} H_{\mathrm{Ar}}\right), 7.86\left(\mathrm{~s}, 1 \mathrm{H}, \mathrm{C} H_{\mathrm{Ar}}\right), 7.79-7.82(\mathrm{~m}, 3 \mathrm{H}$, $\left.\mathrm{CH}_{\mathrm{Ar}}\right), 7.47-7.39\left(\mathrm{~m}, 6 \mathrm{H}, \mathrm{CH}_{\mathrm{Ar}}\right), 7.32-7.28\left(\mathrm{~m}, 1 \mathrm{H}, \mathrm{C} H_{\mathrm{Ar}}\right), 7.08\left(\mathrm{~d}, J_{\mathrm{HH}}\right.$ $\left.=7.8 \mathrm{~Hz}, 2 \mathrm{H}, \mathrm{CH}_{\mathrm{Ts}}\right), 5.75\left(\mathrm{~d}, J_{\mathrm{PH}}=6.3 \mathrm{~Hz}, 2 \mathrm{H}, \mathrm{P}(\mathrm{O}) \mathrm{CH}_{2}\right), 2.27(\mathrm{~s}, 3 \mathrm{H}$,
$\left.\mathrm{CH}_{3 \mathrm{Ts}}\right) ;{ }^{31} \mathrm{P}\left\{{ }^{1} \mathrm{H}\right\} \mathrm{NMR}\left(162 \mathrm{MHz}, \mathrm{CDCl}_{3}, 25{ }^{\circ} \mathrm{C}\right): \delta 27.1(\mathrm{~s}) ;{ }^{13} \mathrm{C}\left\{{ }^{1} \mathrm{H}\right\}$ NMR (101 MHz, $\left.\mathrm{CDCl}_{3}, 25^{\circ} \mathrm{C}\right): \delta 148.8$ (s, $C \mathrm{H}_{\mathrm{Py}}$ ), 145.7 (s, $\left.C_{\mathrm{Py}}\right), 143.3$ $\left(\mathrm{s}, C_{\mathrm{Ts}}\right), 140.5\left(\mathrm{~s}, C \mathrm{H}_{\mathrm{Py}}\right), 139.5\left(\mathrm{~s}, C_{\mathrm{Ts}}\right), 135.6\left(\mathrm{~d}, J_{\mathrm{PC}}=2.6 \mathrm{~Hz}, C \mathrm{H}_{\mathrm{Im}-2}\right)$, $133.0\left(\mathrm{~d}, J_{\mathrm{PC}}=2.6 \mathrm{~Hz}, C \mathrm{H}_{\mathrm{Ph}}\right), 131.3\left(\mathrm{~d}, J_{\mathrm{PC}}=9.9 \mathrm{~Hz}, C \mathrm{H}_{\mathrm{Ph}}\right), 129.2(\mathrm{~d}$, $\left.J_{\mathrm{PC}}=12.5 \mathrm{~Hz}, C \mathrm{H}_{\mathrm{Ph}}\right), 128.7\left(\mathrm{~s}, C \mathrm{H}_{\mathrm{Ts}}\right), 127.9\left(\mathrm{~d}, J_{\mathrm{PC}}=103.6 \mathrm{~Hz}, C_{i p s o}\right.$ Ph), 125.9 (s, $\left.C \mathrm{H}_{\mathrm{Ts}}\right), 125.0$ (s, $\left.C \mathrm{HPy}_{\mathrm{Py}}\right), 124.3$ (s, $\left.C \mathrm{HPy}_{\mathrm{Py}}\right), 118.6$ (s, $C \mathrm{H}_{\mathrm{Im}}$

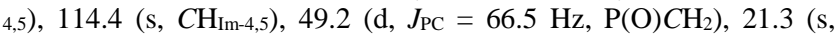
$\mathrm{CH}_{3 \mathrm{Ts}}$ ); Anal. Found: $\mathrm{C}, 61.26 ; \mathrm{H}, 4.87 ; \mathrm{N}, 7.76$. Calcd. $(\%)$ for $\mathrm{C}_{28} \mathrm{H}_{26} \mathrm{~N}_{3} \mathrm{O}_{4}$ PS. $0.25\left(\mathrm{CH}_{2} \mathrm{Cl}_{2}\right)$ : C, 61.38; H, 4.83; N, 7.60; MS $\left(\mathrm{ES}^{+}\right)$: $m / z: 360.1[\mathrm{M}]^{+}$; HRMS $\left(\mathrm{ES}^{+}\right)$: calcd. for $\mathrm{C}_{21} \mathrm{H}_{19} \mathrm{~N}_{3} \mathrm{OP}^{+}$360.1266; found, $360.1266\left(\varepsilon_{\mathrm{r}}=0.0 \mathrm{ppm}\right)$.

Synthesis of [ $\left.\mathbf{P h}_{2} \mathbf{P C H}_{2} \mathbf{I m P y}\right]$ OTs [7](OTs). A dried Schlenk tube containing a stirring bar was charged with bis(4-nitrophenyl) phosphate (128 mg, $0.38 \mathrm{mmol})$ and [6](OTs) $(2.0 \mathrm{~g}, 3.8 \mathrm{mmol})$ under argon flow. Dry toluene $(40 \mathrm{~mL})$ and $\mathrm{PhSiH}_{3}(1.9 \mathrm{~mL}, 15 \mathrm{mmol})$ were added and the mixture was stirred at $140{ }^{\circ} \mathrm{C}$ for $16 \mathrm{~h}$, cooled to RT and the volatiles were removed under vacuum. The resulting solid was dissolved in degassed $\mathrm{CH}_{2} \mathrm{Cl}_{2}(5 \mathrm{~mL})$ and then degassed $\mathrm{Et}_{2} \mathrm{O}(50 \mathrm{~mL})$ was added. The resulting precipitate was washed with degassed $\mathrm{Et}_{2} \mathrm{O}(20 \mathrm{~mL})$ and dried under vacuum to afford [7](OTs) as a beige powder $(1.7 \mathrm{~g}, 89 \%)$. ${ }^{1} \mathrm{H}$ NMR $\left(600 \mathrm{MHz}, \mathrm{CDCl}_{3}, 25^{\circ} \mathrm{C}\right): \delta 10.59\left(\mathrm{~s}, 1 \mathrm{H}, \mathrm{CH}_{\mathrm{Im}-2}\right), 8.40(\mathrm{~d}, J$ $\left.=4.5 \mathrm{~Hz}, 1 \mathrm{H}, \mathrm{C} H_{\mathrm{Ar}}\right), 8.26\left(\mathrm{~d}, J=8.2 \mathrm{~Hz}, 1 \mathrm{H}, \mathrm{C} H_{\mathrm{Ar}}\right), 8.08\left(\mathrm{~s}, 1 \mathrm{H}, \mathrm{CH}_{\mathrm{Ar}}\right)$, $7.90-7.88\left(\mathrm{~m}, 1 \mathrm{H}, \mathrm{C} H_{\mathrm{Ar}}\right), 7.79\left(\mathrm{~d}, J_{\mathrm{HH}}=7.8 \mathrm{~Hz}, 2 \mathrm{H}, \mathrm{C} H_{\mathrm{Ts}}\right), 7.54-7.51$ $\left(\mathrm{m}, 4 \mathrm{H}, \mathrm{CH}_{\mathrm{Ar}}\right), 7.37-7.32\left(\mathrm{~m}, 7 \mathrm{H}, \mathrm{C} H_{\mathrm{Ar}}\right), 7.22\left(\mathrm{~s}, 1 \mathrm{H}, \mathrm{C} H_{\mathrm{Ar}}\right), 7.09(\mathrm{~d}$, $\left.J_{\mathrm{HH}}=7.8 \mathrm{~Hz}, 2 \mathrm{H}, \mathrm{C} H_{\mathrm{Ts}}\right), 5.34\left(\mathrm{~d}, J_{\mathrm{PH}}=5.3 \mathrm{~Hz}, 2 \mathrm{H}, \mathrm{P}(\mathrm{O}) \mathrm{CH}_{2}\right), 2.30(\mathrm{~s}$, $\left.3 \mathrm{H}, \mathrm{CH}_{3 \mathrm{Ts}}\right) ;{ }^{31} \mathrm{P}\left\{{ }^{1} \mathrm{H}\right\} \mathrm{NMR}\left(162 \mathrm{MHz} \mathrm{CDCl}_{3}, 25{ }^{\circ} \mathrm{C}\right): \delta-11.1(\mathrm{~s})$; ${ }^{13} \mathrm{C}\left\{{ }^{1} \mathrm{H}\right\}$ NMR $\left(151 \mathrm{MHz}, \mathrm{CDCl}_{3}, 25^{\circ} \mathrm{C}\right): \delta 148.9$ (s, $\left.C \mathrm{H}_{\mathrm{Py}}\right), 146.0(\mathrm{~s}$, $\left.C_{\mathrm{Py}}\right), 143.6$ (s, $\left.C_{\mathrm{Ts}}\right), 140.8$ (s, $\left.C \mathrm{H}_{\mathrm{Py}}\right), 139.5$ (s, $\left.C_{\mathrm{Ts}}\right), 136.0$ (s, $C_{\mathrm{Im}-2}$ ), $133.4\left(\mathrm{~d}, J_{\mathrm{PC}}=19.8 \mathrm{~Hz}, C \mathrm{HPh}\right), 132.9\left(\mathrm{~d}, J_{\mathrm{PC}}=12.0 \mathrm{~Hz}, C_{i p s o \mathrm{Ph}}\right), 130.3$ $\left(\mathrm{s}, C \mathrm{HPh}_{\mathrm{Ph}}\right), 129.3\left(\mathrm{~d}, J_{\mathrm{PC}}=7.3 \mathrm{~Hz}, C \mathrm{HPh}_{\mathrm{Ph}}\right), 128.8\left(\mathrm{~s}, C \mathrm{H}_{\mathrm{Ts}}\right), 126.1(\mathrm{~s}$,

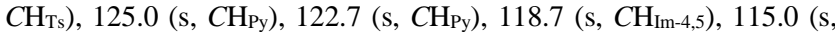
$C_{\mathrm{Im}-4,5)}, 49.7\left(\mathrm{~d}, J_{\mathrm{PC}}=21.9 \mathrm{~Hz}, \mathrm{PCH}_{2}\right), 21.4\left(\mathrm{~s}, \mathrm{CH}_{3 \mathrm{Ts}}\right)$; Anal. Found: C, 61.43; H, 4.91; N, 7.31. Calcd. (\%) for $\mathrm{C}_{28} \mathrm{H}_{26} \mathrm{~N}_{3} \mathrm{O}_{3}$ PS. 0.50 $\left(\mathrm{CH}_{2} \mathrm{Cl}_{2}\right): \mathrm{C}, 61.34 ; \mathrm{H}, 4.88 ; \mathrm{N}, 7.53 ; \mathrm{MS}\left(\mathrm{ES}^{+}\right): \mathrm{m} / z: 344.1[\mathrm{M}]^{+}$; HRMS (ES ${ }^{+}$): calcd. for $\mathrm{C}_{21} \mathrm{H}_{19} \mathrm{~N}_{3} \mathrm{P}^{+} 344.1317$; found, $344.1317\left(\varepsilon_{\mathrm{r}}=\right.$ $0.0 \mathrm{ppm})$.

Synthesis of [ $\left.\mathrm{Ph}_{2} \mathbf{P}(\mathrm{O}) \mathrm{CH}_{2} \mathbf{I m C H} \mathrm{H}_{2} \mathrm{Py}\right] \mathrm{OTs}$ [9](OTs). A mixture of 8 (1.98 g, $12.4 \mathrm{mmol})$ and $\mathrm{Ph}_{2} \mathrm{P}(\mathrm{O}) \mathrm{CH}_{2} \mathrm{OTs}(4.00 \mathrm{~g}, 10.4 \mathrm{mmol})$ was heated under stirring at $140{ }^{\circ} \mathrm{C}$ for $16 \mathrm{~h}$. After cooling to RT, the brown solid formed was dissolved in $\mathrm{CH}_{2} \mathrm{Cl}_{2}(5 \mathrm{~mL})$ and then $\mathrm{Et}_{2} \mathrm{O}(100 \mathrm{~mL})$ was added. The resulting precipitate was triturated with $\mathrm{Et}_{2} \mathrm{O}(30 \mathrm{~mL})$ until the formation of a white/brown powder ( $c a .30 \mathrm{~min})$. The supernatant was removed by decantation and the precipitate was washed with $\mathrm{Et}_{2} \mathrm{O}(10 \mathrm{~mL})$ and dried under vacuum to afford [9](OTs) $(4.8 \mathrm{~g}, 87 \%)$ as very hygroscopic brown crystals. ${ }^{1} \mathrm{H}$ NMR $\left(400 \mathrm{MHz}, \mathrm{CDCl}_{3}, 25\right.$ $\left.{ }^{\circ} \mathrm{C}\right): \delta 9.95\left(\mathrm{~s}, 1 \mathrm{H}, \mathrm{CH}_{\mathrm{Im}-2}\right), 8.43\left(\mathrm{~d}, J=4.8 \mathrm{~Hz}, 1 \mathrm{H}, \mathrm{CH}_{\mathrm{Ar}}\right), 7.97-7.88$ $\left(\mathrm{m}, 4 \mathrm{H}, \mathrm{C} H_{\mathrm{Ar}}\right), 7.79\left(\mathrm{~d}, J_{\mathrm{HH}}=7.7 \mathrm{~Hz}, 2 \mathrm{H}, \mathrm{C} H_{\mathrm{Ts}}\right), 7.65\left(\mathrm{~s}, 1 \mathrm{H}, \mathrm{CH}_{\mathrm{Ar}}\right)$, 7.63-7.58 (m, $\left.1 \mathrm{H}, \mathrm{CH}_{\mathrm{Ar}}\right), 7.51-7.45\left(\mathrm{~m}, 2 \mathrm{H}, \mathrm{CH}_{\mathrm{Ar}}\right), 7.43-7.35(\mathrm{~m}, 4 \mathrm{H}$, $\left.\mathrm{CH}_{\mathrm{Ar}}\right), 7.36-7.29\left(\mathrm{~m}, 2 \mathrm{H}, \mathrm{C} H_{\mathrm{Ar}}\right), 7.24-7.19\left(\mathrm{~m}, 1 \mathrm{H}, \mathrm{C} H_{\mathrm{Ar}}\right), 7.12\left(\mathrm{~d}, J_{\mathrm{HH}}\right.$ $\left.=7.8 \mathrm{~Hz}, 2 \mathrm{H}, \mathrm{C} H_{\mathrm{Ts}}\right), 5.46\left(\mathrm{~d}, J_{\mathrm{PH}}=6.2 \mathrm{~Hz}, 2 \mathrm{H}, \mathrm{P}(\mathrm{O}) \mathrm{CH}_{2}\right), 5.33(\mathrm{~s}, 2 \mathrm{H}$, $\left.\mathrm{PyCH}_{2}\right), 2.32$ (s, 3H, $\left.\mathrm{CH}_{3 \mathrm{Ts}}\right) ;{ }^{31} \mathrm{P}\left\{{ }^{1} \mathrm{H}\right\}$ NMR $\left(162 \mathrm{MHz}, \mathrm{CDCl}_{3}, 25{ }^{\circ} \mathrm{C}\right)$ : $\delta 27.2(\mathrm{~s}) ;{ }^{13} \mathrm{C}\left\{{ }^{1} \mathrm{H}\right\}$ NMR $\left(101 \mathrm{MHz}, \mathrm{CDCl}_{3}, 25{ }^{\circ} \mathrm{C}\right): \delta 152.3\left(\mathrm{~s}, C_{\mathrm{Py}}\right)$, $149.9\left(\mathrm{~s}, C_{\mathrm{Py}}\right), 143.5\left(\mathrm{~s}, C_{\mathrm{Ts}}\right), 139.6\left(\mathrm{~s}, C_{\mathrm{Ts}}\right), 138.5\left(\mathrm{~d}, J_{\mathrm{PC}}=2.3 \mathrm{~Hz}\right.$, $\left.C \mathrm{H}_{\mathrm{Im}-2}\right), 137.7\left(\mathrm{~s}, C \mathrm{H}_{\mathrm{Py}}\right), 133.1\left(\mathrm{~d}, J_{\mathrm{PC}}=2.3 \mathrm{~Hz}, C \mathrm{H}_{\mathrm{Ph}}\right), 131.4\left(\mathrm{~d}, J_{\mathrm{PC}}=\right.$ $\left.10.0 \mathrm{~Hz}, C \mathrm{H}_{\mathrm{Ph}}\right), 129.3\left(\mathrm{~d}, J_{\mathrm{PC}}=12.5 \mathrm{~Hz}, C \mathrm{H}_{\mathrm{Ph}}\right), 128.8\left(\mathrm{~s}, C \mathrm{H}_{\mathrm{Ts}}\right), 127.9$ $\left(\mathrm{d}, J_{\mathrm{PC}}=103.3 \mathrm{~Hz}, C_{i p s o} \mathrm{Ph}\right), 126.0\left(\mathrm{~s}, C \mathrm{H}_{\mathrm{Ts}}\right), 124.0\left(\mathrm{~s}, C \mathrm{H}_{\mathrm{Py}}\right), 123.5$ (s,

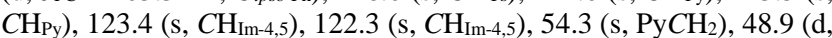
$\left.J_{\mathrm{PC}}=66.0 \mathrm{~Hz}, \mathrm{P}(\mathrm{O}) C \mathrm{CH}_{2}\right), 21.4\left(\mathrm{~s}, \mathrm{CH}_{3 \mathrm{Ts}}\right)$; Anal. Found: $\mathrm{C}, 61.20 ; \mathrm{H}$, 5.02; N, 7.64. Calcd. (\%) for $\mathrm{C}_{29} \mathrm{H}_{28} \mathrm{~N}_{3} \mathrm{O}_{4}$ PS. $0.35\left(\mathrm{CH}_{2} \mathrm{Cl}_{2}\right)$ : C, 61.27; H, 5.03; N, 7.30; MS (ES+): m/z: $374.1\left[\mathrm{M}^{+}\right.$; HRMS $\left(\mathrm{ES}^{+}\right)$: calcd. for $\mathrm{C}_{22} \mathrm{H}_{21} \mathrm{~N}_{3} \mathrm{OP}^{+} 374.1422$; found, $374.1431\left(\varepsilon_{\mathrm{r}}=2.4 \mathrm{ppm}\right)$.

Synthesis of $\left[\mathrm{Ph}_{2} \mathbf{P C H}_{2} \mathbf{I m C H} \mathrm{H}_{2} \mathbf{P y}\right] \mathbf{O T s}[10](\mathrm{OTs})$. A Schlenk tube was charged with bis(4-nitrophenyl) phosphate $(180 \mathrm{mg}, 0.53 \mathrm{mmol})$ and [9](OTs) $(2.8 \mathrm{~g}, 5.3 \mathrm{mmol})$ under argon flow. Dry toluene $(30 \mathrm{~mL})$ and $\mathrm{PhSiH}_{3}(2.6 \mathrm{~mL}, 21 \mathrm{mmol})$ were added and the mixture was stirred at $110{ }^{\circ} \mathrm{C}$ for $16 \mathrm{~h}$. After cooling to RT, the volatiles were removed under vacuum. The solid residue was dissolved in degassed $\mathrm{CH}_{2} \mathrm{Cl}_{2}(5$ $\mathrm{mL})$ and then degassed $\mathrm{Et}_{2} \mathrm{O}$ was added $(50 \mathrm{~mL})$. The precipitate formed was washed with degassed $\mathrm{Et}_{2} \mathrm{O}(20 \mathrm{~mL})$ and dried under vacuum to afford [10](OTs) $(2.4 \mathrm{~g}, 86 \%)$ as very hygroscopic brown crystals. ${ }^{1} \mathrm{H}$ NMR $\left(400 \mathrm{MHz}, \mathrm{CDCl}_{3}, 25{ }^{\circ} \mathrm{C}\right): \delta 9.56\left(\mathrm{~s}, 1 \mathrm{H}, \mathrm{C} H_{\mathrm{Im}-2}\right), 8.37$ 
$\left(\mathrm{d}, J=4.8 \mathrm{~Hz}, 1 \mathrm{H}, \mathrm{C} H_{\mathrm{Ar}}\right), 7.74\left(\mathrm{~d}, J_{\mathrm{HH}}=7.7 \mathrm{~Hz}, 2 \mathrm{H}, \mathrm{C} H_{\mathrm{Ts}}\right), 7.55-7.52$ $\left(\mathrm{m}, 1 \mathrm{H}, \mathrm{C} H_{\mathrm{Ar}}\right), 7.44\left(\mathrm{~s}, 1 \mathrm{H}, \mathrm{C} H_{\mathrm{Ar}}\right), 7.39-7.36\left(\mathrm{~m}, 5 \mathrm{H}, \mathrm{C} H_{\mathrm{Ar}}\right), 7.30-7.20$ $\left(\mathrm{m}, 6 \mathrm{H}, \mathrm{C} H_{\mathrm{Ar}}\right), 7.18-7.10\left(\mathrm{~m}, 2 \mathrm{H}, \mathrm{CH}_{\mathrm{Ar}}\right), 7.04\left(\mathrm{~d}, J_{\mathrm{HH}}=7.7 \mathrm{~Hz}, 2 \mathrm{H}\right.$, $\left.\mathrm{CH}_{\mathrm{Ts}}\right), 5.36\left(\mathrm{~s}, 2 \mathrm{H}, \mathrm{PyCH}_{2}\right), 4.95\left(\mathrm{~d}, J_{\mathrm{PH}}=5.4 \mathrm{~Hz}, 2 \mathrm{H}, \mathrm{PCH}\right), 2.26(\mathrm{~s}$, $\left.3 \mathrm{H}, \mathrm{CH}_{3 \mathrm{Ts}}\right) ;{ }^{31} \mathrm{P}\left\{{ }^{1} \mathrm{H}\right\}$ NMR $\left(162 \mathrm{MHz}, \mathrm{CDCl}_{3}, 25{ }^{\circ} \mathrm{C}\right): \delta-12.2(\mathrm{~s})$; ${ }^{13} \mathrm{C}\left\{{ }^{1} \mathrm{H}\right\}$ NMR $\left(101 \mathrm{MHz}, \mathrm{CDCl}_{3}, 25{ }^{\circ} \mathrm{C}\right): \delta 152.6\left(\mathrm{~s}, C_{\mathrm{Py}}\right), 149.5(\mathrm{~s}$, $\left.C \mathrm{H}_{\mathrm{Py}}\right), 143.9$ (s, $\left.C_{\mathrm{Ts}}\right), 139.0\left(\mathrm{~s}, C_{\mathrm{Ts}}\right), 137.5\left(\mathrm{~s}, C \mathrm{H}_{\mathrm{Im}-2}\right), 137.3$ (s, $\left.C \mathrm{H}_{\mathrm{Py}}\right)$, $133.0\left(\mathrm{~d}, J_{\mathrm{PC}}=19.4 \mathrm{~Hz}, C \mathrm{HPh}_{\mathrm{Ph}}, 132.9\left(\mathrm{~d}, J_{\mathrm{PC}}=11.4 \mathrm{~Hz}, C_{\text {ipso }} \mathrm{Ph}\right), 129.9\right.$ $\left(\mathrm{s}, C \mathrm{H}_{\mathrm{Ph}}\right), 128.9\left(\mathrm{~d}, J_{\mathrm{PC}}=7.4 \mathrm{~Hz}, C \mathrm{H}_{\mathrm{Ph}}\right), 128.5\left(\mathrm{~s}, C \mathrm{H}_{\mathrm{Ts}}\right), 125.9(\mathrm{~s}$, CH $\mathrm{H}_{\mathrm{Ts}}$ ), 123.5 (s, $C \mathrm{H}_{\mathrm{Py}}$ ), 123.4 (s, $C \mathrm{HPy}_{\mathrm{Py}}$ ), 122.7 (s, $C \mathrm{H}_{\mathrm{Im}-4,5),} 121.9$ (s, $\left.C \mathrm{H}_{\mathrm{Im}-4,5)}\right), 53.7\left(\mathrm{~s}, \mathrm{PyCH}_{2}\right), 48.7\left(\mathrm{~d}, J_{\mathrm{PC}}=22.1 \mathrm{~Hz}, \mathrm{PCH}_{2}\right), 21.2(\mathrm{~s}$, $\mathrm{CH}_{3 \mathrm{Ts}}$ ); Anal. Found: $\mathrm{C}, 63.25 ; \mathrm{H}, 5.06 ; \mathrm{N}, 8.15$. Calcd. (\%) for $\mathrm{C}_{29} \mathrm{H}_{28} \mathrm{~N}_{3} \mathrm{O}_{3}$ PS. 0.3 $\mathrm{CH}_{2} \mathrm{Cl}_{2}$ : C, 63.40; H, 5.19; N, 7.57; MS (ES $\left.{ }^{+}\right): m / z:$ $358.1[\mathrm{M}]^{+}$; HRMS $\left(\mathrm{ES}^{+}\right)$: calcd. for $\mathrm{C}_{22} \mathrm{H}_{21} \mathrm{~N}_{3} \mathrm{P}^{+} 358.1473$; found, $358.1482\left(\varepsilon_{\mathrm{r}}=2.5 \mathrm{ppm}\right)$

Synthesis of PCP Bidentate Complex 11. To a stirred suspension of $[4](\mathrm{OTs})(0.40 \mathrm{~g}, 0.63 \mathrm{mmol})$ in toluene $(30 \mathrm{~mL})$, a solution of KHMDS (0.5 M in toluene, $1.3 \mathrm{~mL}, 0.66 \mathrm{mmol})$ was added at RT and then the resulting yellow suspension was sonicated during $5 \mathrm{~min}$ and stirred for additional $15 \mathrm{~min}$. Solid $\left[\mathrm{Mn}(\mathrm{CO})_{5} \mathrm{Br}\right](0.17 \mathrm{~g}, 0.63 \mathrm{mmol})$ was added in one portion inducing a vigorous $\mathrm{CO}$ evolution. The resulting orange solution was then stirred for $2 \mathrm{~h}$ at $60{ }^{\circ} \mathrm{C}$. After cooling the reaction mixture to RT, filtration through Celite and evaporation of the solvent under vacuum, the crude product was crystallized in a THF/pentane mixture to afford $\mathbf{1 1}$ as a yellow powder $(0.32 \mathrm{~g}, 71 \%)$. ${ }^{1} \mathrm{H}$ NMR $\left(400 \mathrm{MHz}, \mathrm{CD}_{2} \mathrm{Cl}_{2}, 25^{\circ} \mathrm{C}\right): \delta 7.93\left(\mathrm{t}, J=8.7 \mathrm{~Hz}, 2 \mathrm{H}, \mathrm{C} H_{\mathrm{Ph}}\right)$, 7.56-7.35 (m, 16H, CH $\left.H_{\mathrm{Ph}}\right), 7.21-7.13\left(\mathrm{~m}, 3 \mathrm{H}, \mathrm{C} H_{\mathrm{Ph}}\right), 6.99$ (brs, $1 \mathrm{H}$,

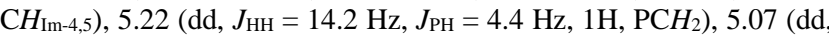
$\left.J_{\mathrm{HH}}=14.2 \mathrm{~Hz}, J_{\mathrm{PH}}=3.5 \mathrm{~Hz}, 1 \mathrm{H}, \mathrm{PCH}_{2}\right), 4.88-4.98\left(\mathrm{~m}, 2 \mathrm{H}, \mathrm{PCH}_{2}\right)$; ${ }^{31} \mathrm{P}\left\{{ }^{1} \mathrm{H}\right\}$ NMR (162 MHz, $\left.\mathrm{CD}_{2} \mathrm{Cl}_{2}, 25{ }^{\circ} \mathrm{C}\right): \delta 71.0(\mathrm{~s}),-17.0(\mathrm{~s}) ;$ ${ }^{13} \mathrm{C}\left\{{ }^{1} \mathrm{H}\right\}$ NMR $\left(101 \mathrm{MHz}, \mathrm{CD}_{2} \mathrm{Cl}_{2}, 25{ }^{\circ} \mathrm{C}\right): \delta 222.9\left(\mathrm{~d}, J_{\mathrm{PC}}=17.8 \mathrm{~Hz}\right.$, $C O), 221.4\left(\mathrm{~d}, J_{\mathrm{PC}}=31.0 \mathrm{~Hz}, C \mathrm{C}\right), 220.1\left(\mathrm{~d}, J_{\mathrm{PC}}=21.5 \mathrm{~Hz}, C \mathrm{C}\right), 197.1$ $\left(\mathrm{d}, J_{\mathrm{PC}}=16.5 \mathrm{~Hz}, C \mathrm{~N}_{2}\right), 135.6\left(\mathrm{~d}, J_{\mathrm{PC}}=3.0 \mathrm{~Hz}, C_{i p s o} \mathrm{Ph}\right), 135.4\left(\mathrm{~d}, J_{\mathrm{PC}}=\right.$ $\left.21.2 \mathrm{~Hz}, C_{i p s o} \mathrm{Ph}\right), 135.2\left(\mathrm{~d}, J_{\mathrm{PC}}=8.1 \mathrm{~Hz}, C_{i p s o} \mathrm{Ph}\right), 134.7\left(\mathrm{~d}, J_{\mathrm{PC}}=9.9\right.$ $\left.\mathrm{Hz}, C \mathrm{H}_{\mathrm{Ph}}\right), 133.6\left(\mathrm{~d}, J_{\mathrm{PC}}=19.8 \mathrm{~Hz}, C \mathrm{H}_{\mathrm{Ph}}\right), 133.2\left(\mathrm{~d}, J_{\mathrm{PC}}=19.1 \mathrm{~Hz}\right.$, $\left.C \mathrm{H}_{\mathrm{Ph}}\right), 131.8$ (s, $\left.C \mathrm{H}_{\mathrm{Ph}}\right), 130.6\left(\mathrm{~d}, J_{\mathrm{PC}}=10.1 \mathrm{~Hz}, C \mathrm{H}_{\mathrm{Ph}}\right), 130.5\left(\mathrm{~s}, C \mathrm{H}_{\mathrm{Ph}}\right)$, 130.1 (s, $\left.C \mathrm{H}_{\mathrm{Ph}}\right), 129.9$ (s, $\left.C \mathrm{H}_{\mathrm{Ph}}\right), 129.4\left(\mathrm{~s}, C \mathrm{H}_{\mathrm{Ph}}\right), 129.2\left(\mathrm{~d}, J_{\mathrm{PC}}=6.1\right.$

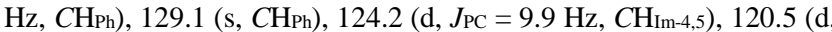

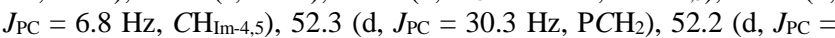
$17.2 \mathrm{~Hz}, \mathrm{PCH}_{2}$ ); IR (toluene): $v_{\mathrm{CO}} 2015$ (s), 1940 (s), 1910 (s) $\mathrm{cm}^{-1}$; Anal. Found: C, 54.50; H, 3.33; N, 4.13. Calcd. (\%) for $\mathrm{C}_{32} \mathrm{H}_{26} \mathrm{BrMnN}_{2} \mathrm{O}_{3} \mathrm{P}_{2} .0 .35\left(\mathrm{CH}_{2} \mathrm{Cl}_{2}\right): \mathrm{C}, 54.49 ; \mathrm{H}, 3.77 ; \mathrm{N}, 3.93 ; \mathrm{MS}$ $\left(\mathrm{ES}^{+}\right): \quad m / z: \quad 603.1 \quad\left[\mathrm{M}-\mathrm{Br}^{+} ; \mathrm{HRMS} \quad\left(\mathrm{ES}^{+}\right)\right.$: calcd. for $\mathrm{C}_{32} \mathrm{H}_{26} \mathrm{MnN}_{2} \mathrm{O}_{3} \mathrm{P}_{2}{ }^{+}$603.0799; found, $603.0799\left(\varepsilon_{\mathrm{r}}=0.0 \mathrm{ppm}\right)$.

Synthesis of PCP Tridentate Complex [12](OTf). To a solution of $11(0.4 \mathrm{~g}, 0.58 \mathrm{mmol})$ in $\mathrm{CH}_{2} \mathrm{Cl}_{2}(30 \mathrm{~mL}) \operatorname{AgOTf}(0.17 \mathrm{~g}, 0.66 \mathrm{mmol})$ was added as a solid. The reaction mixture was then stirred at RT for 2 h. After filtration through Celite and evaporation of the solvent under vacuum, $[\mathbf{1 2}]$ (OTf) was obtained as a pale-yellow powder $(0.39 \mathrm{~g}$, $88 \%$ ). Single crystals suitable for XRD were obtained by slow evaporation of a $\mathrm{CH}_{2} \mathrm{Cl}_{2}$ /toluene solution at $\mathrm{RT}$. ${ }^{1} \mathrm{H} \mathrm{NMR}\left(400 \mathrm{MHz}, \mathrm{CD}_{2} \mathrm{Cl}_{2}\right.$, $\left.25^{\circ} \mathrm{C}\right): \delta 7.82$ (brs, $\left.2 \mathrm{H}, \mathrm{CH}_{\mathrm{Ar}}\right), 7.58-7.50\left(\mathrm{~m}, 20 \mathrm{H}, \mathrm{C} H_{\mathrm{Ar}}\right), 5.26-5.24$ $\left(\mathrm{m}, 4 \mathrm{H}, \mathrm{PCH} \mathrm{H}_{2}\right) ;{ }^{31} \mathrm{P}\left\{{ }^{1} \mathrm{H}\right\}$ NMR $\left(162 \mathrm{MHz}, \mathrm{CD}_{2} \mathrm{Cl}_{2}, 25^{\circ} \mathrm{C}\right): \delta 89.6(\mathrm{~s}) ;$ ${ }^{13} \mathrm{C}\left\{{ }^{1} \mathrm{H}\right\}$ NMR $\left(101 \mathrm{MHz}, \mathrm{CD}_{2} \mathrm{Cl}_{2}, 25{ }^{\circ} \mathrm{C}\right): \delta 220.7$ (brt, $J_{\mathrm{PC}}=10.5 \mathrm{~Hz}$, $C O), 213.6\left(\right.$ brt, $\left.J_{\mathrm{PC}}=19.0 \mathrm{~Hz}, C \mathrm{O}\right), 195.6\left(\mathrm{t}, J_{\mathrm{PC}}=13.1 \mathrm{~Hz}, C \mathrm{~N}_{2}\right)$, $133.8\left(\mathrm{~m}, C_{\text {ipso }} \mathrm{Ph}\right) 132.2$ (s, $\left.C \mathrm{H}_{\mathrm{Ph}}\right), 131.7-132.0\left(\mathrm{~m}, C \mathrm{H}_{\mathrm{Ph}}\right), 129.8-130.2$ $\left(\mathrm{m}, C \mathrm{H}_{\mathrm{Ph}}\right), 124.9\left(\mathrm{~s}, C_{\mathrm{Im}-4,5}\right), 121.6\left(\mathrm{q}, J_{\mathrm{CF}}=321.4 \mathrm{~Hz}, \mathrm{CF}_{3}\right), 56.2(\mathrm{~m}$, $\mathrm{PCH}_{2}$ ); IR (THF): v $v_{\mathrm{CO}} 2045$ (w), 1966 (vs), 1942 (s) cm ${ }^{-1}$; Anal. Found: C, 52.34; $\mathrm{H}, 3.82 ; \mathrm{N}, 3.60$. Calcd. (\%) for $\mathrm{C}_{33} \mathrm{H}_{26} \mathrm{~F}_{3} \mathrm{MnN}_{2} \mathrm{O}_{6} \mathrm{P} 2 \mathrm{~S}$ : C, 52.67; H, 3.48; N, 3.72; MS $\left(\mathrm{ES}^{+}\right): m / z: 603.1[\mathrm{M}]^{+}$; HRMS $\left(\mathrm{ES}^{+}\right)$: calcd. for $\mathrm{C}_{32} \mathrm{H}_{26} \mathrm{MnN}_{2} \mathrm{O}_{3} \mathrm{P}_{2}{ }^{+}$603.0799; found, $603.0816\left(\varepsilon_{\mathrm{r}}=2.8 \mathrm{ppm}\right)$.

Synthesis of PCN Bidentate Complexes 13 and 14. To a stirred suspension of [7](OTs) $(0.20 \mathrm{~g}, 0.39 \mathrm{mmol})$ in THF $(30 \mathrm{~mL})$, a solution of KHMDS $(0.5 \mathrm{M}$ in Toluene, $0.9 \mathrm{~mL}, 0.43 \mathrm{mmol})$ was added at RT and then the resulting brown suspension was sonicated during $5 \mathrm{~min}$ and stirred for additional $5 \mathrm{~min}$. Solid $\left[\mathrm{Mn}(\mathrm{CO})_{5} \mathrm{Br}\right](0.11 \mathrm{~g}, 0.39$ $\mathrm{mmol}$ ) was added in one portion inducing a vigorous $\mathrm{CO}$ evolution. The resulting orange solution was then stirred for $15 \mathrm{~min}$ at RT. After filtration through Celite and evaporation of the solvent under vacuum, a mixture of two complexes $\mathbf{1 3}$ and $\mathbf{1 4}$ in a 1:3 ratio was obtained as yellow-orange powder $(0.16 \mathrm{~g}, 80 \%)$. The crude residue was recrystallized in $\mathrm{THF} / \mathrm{Et}_{2} \mathrm{O}$ mixture affording complex 14 as a yellow-orange powder $(0.11 \mathrm{~g}, 51 \%)$. Single crystals for both complexes suitable for XRD were obtained by slow evaporation of $\mathrm{CH}_{2} \mathrm{Cl}_{2}$ solutions at RT. MS $\left(\mathrm{ES}^{+}\right): \quad m / z: 562.0 \quad[\mathrm{M}+\mathrm{H}]^{+} ; \quad \mathrm{HRMS} \quad\left(\mathrm{ES}^{+}\right)$: calcd. for $\mathrm{C}_{24} \mathrm{H}_{19} \mathrm{MnBrN}_{3} \mathrm{O}_{3} \mathrm{P}^{+}$561.9728; found, 561.9730 ( $\left.\varepsilon_{\mathrm{r}}=0.4 \mathrm{ppm}\right)$.

The NMR and IR data of complex 13 were deduced from the 13/14 mixture. 13: ${ }^{1} \mathrm{H}$ NMR $\left(400 \mathrm{MHz}, \mathrm{CD}_{2} \mathrm{Cl}_{2}\right): \delta 8.67(\mathrm{~d}, J=4.0 \mathrm{~Hz}, 1 \mathrm{H}$, $\left.\mathrm{C}_{\mathrm{Ar}}\right), 7.98-7.94\left(\mathrm{~m}, 3 \mathrm{H}, \mathrm{C} H_{\mathrm{Ar}}\right), 7.61-7.24\left(\mathrm{~m}, 12 \mathrm{H}, \mathrm{C} H_{\mathrm{Ar}}\right), 5.11(\mathrm{t}, J$ $\left.=12.0 \mathrm{~Hz}, 1 \mathrm{H}, \mathrm{PCH} \mathrm{H}_{2}\right), 4.97\left(\mathrm{~d}, J=12.0 \mathrm{~Hz}, 1 \mathrm{H}, \mathrm{PCH}{ }_{2}\right) ;{ }^{31} \mathrm{P}\left\{{ }^{1} \mathrm{H}\right\} \mathrm{NMR}$ $\left(162 \mathrm{MHz}, \mathrm{CD}_{2} \mathrm{Cl}_{2}, 25{ }^{\circ} \mathrm{C}\right): \delta 71.8(\mathrm{~s}) ;{ }^{13} \mathrm{C}\left\{{ }^{1} \mathrm{H}\right\}$ NMR $(101 \mathrm{MHz}$, $\left.\mathrm{CD}_{2} \mathrm{Cl}_{2}\right): \delta 223.9\left(\mathrm{~d}, J_{\mathrm{PC}}=18.2 \mathrm{~Hz}, C \mathrm{O}\right), 220.2\left(\mathrm{~d}, J_{\mathrm{PC}}=18.2 \mathrm{~Hz}, C \mathrm{O}\right)$, $218.5\left(\mathrm{~d}, J_{\mathrm{PC}}=32.3 \mathrm{~Hz}, C \mathrm{O}\right), 198.1\left(\mathrm{~d}, J_{\mathrm{PC}}=15.1 \mathrm{~Hz}, C \mathrm{~N}_{2}\right), 153.1(\mathrm{~s}$, $\left.C_{\mathrm{Py}}\right), 150.0\left(\mathrm{~s}, C \mathrm{H}_{\mathrm{Py}}\right), 139.3\left(\mathrm{~s}, C \mathrm{H}_{\mathrm{Py}}\right), 136.1\left(\mathrm{~d}, J_{\mathrm{PC}}=37.4 \mathrm{~Hz}, C_{i p s o} \mathrm{Ph}\right)$, $135.0\left(\mathrm{~d}, J_{\mathrm{PC}}=10.1 \mathrm{~Hz}, C \mathrm{H}_{\mathrm{Ph}}\right), 131.9\left(\mathrm{~d}, J_{\mathrm{PC}}=2.0 \mathrm{~Hz}, C \mathrm{H}_{\mathrm{Ph}}\right), 130.5(\mathrm{~s}$, $\left.C \mathrm{H}_{\mathrm{Ph}}\right), 130.4\left(\mathrm{~d}, J_{\mathrm{PC}}=10.1 \mathrm{~Hz}, C \mathrm{H}_{\mathrm{Ph}}\right), 129.3\left(\mathrm{~d}, J_{\mathrm{PC}}=9.1 \mathrm{~Hz}, C \mathrm{H}_{\mathrm{Ph}}\right)$, $129.2\left(\mathrm{~d}, J_{\mathrm{PC}}=9.1 \mathrm{~Hz}, C \mathrm{H}_{\mathrm{Ph}}\right), 125.2\left(\mathrm{~s}, C \mathrm{H}_{\mathrm{Py}}\right), 125.1\left(\mathrm{~s}, C \mathrm{H}_{\mathrm{Py}}\right), 122.2$ $\left(\mathrm{s}, C \mathrm{H}_{\mathrm{Im}-4,5}\right), 120.9\left(\mathrm{~d}, J_{\mathrm{PC}}=4.0 \mathrm{~Hz} C \mathrm{H}_{\mathrm{Im}-4,5)}\right), 52.2\left(\mathrm{~d}, J_{\mathrm{PC}}=30.3 \mathrm{~Hz}\right.$, $\mathrm{PCH}_{2}$ ); IR (THF): $v_{\mathrm{CO}} 2016$ (s), 1939 (s), 1904 (s) $\mathrm{cm}^{-1}$

14: ${ }^{1} \mathrm{H}$ NMR $\left(400 \mathrm{MHz}, \mathrm{CD}_{2} \mathrm{Cl}_{2}, 25{ }^{\circ} \mathrm{C}\right): \delta 8.97(\mathrm{~d}, J=5.5 \mathrm{~Hz}, 1 \mathrm{H}$, $\left.\mathrm{CH}_{\mathrm{Ar}}\right), 7.94\left(\mathrm{t}, J=8.0 \mathrm{~Hz}, 1 \mathrm{H}, \mathrm{CH}_{\mathrm{Ar}}\right), 7.60-7.48\left(\mathrm{~m}, 6 \mathrm{H}, \mathrm{CH}_{\mathrm{Ar}}\right), 7.45-$ $7.37\left(\mathrm{~m}, 6 \mathrm{H}, \mathrm{C} H_{\mathrm{Ar}}\right), 7.30\left(\mathrm{t}, J=4.0 \mathrm{~Hz}, 1 \mathrm{H}, \mathrm{CH}_{\mathrm{Ar}}\right), 6.83\left(\mathrm{~s}, 1 \mathrm{H}, \mathrm{CH}_{\mathrm{Ar}}\right)$, $5.37\left(\mathrm{dd}, J=14.1,8.2 \mathrm{~Hz}, 1 \mathrm{H}, \mathrm{PCH}_{2}\right), 4.99(\mathrm{dd}, J=14.1,1.8 \mathrm{~Hz}, 1 \mathrm{H}$ $\left.\mathrm{PCH}_{2}\right) ;{ }^{31} \mathrm{P}\left\{{ }^{1} \mathrm{H}\right\}$ NMR $\left(162 \mathrm{MHz}, \mathrm{CD}_{2} \mathrm{Cl}_{2}, 25{ }^{\circ} \mathrm{C}\right): \delta-15.9(\mathrm{~s}) ;{ }^{13} \mathrm{C}\left\{{ }^{1} \mathrm{H}\right\}$ NMR (101 MHz, $\left.\mathrm{CD}_{2} \mathrm{Cl}_{2}, 25^{\circ} \mathrm{C}\right): \delta 226.4(\mathrm{~s}, C \mathrm{O}), 221.3(\mathrm{~s}, \mathrm{CO}), 218.0$ (s, $C \mathrm{O}$ ), 206.0 (s, $C \mathrm{~N}_{2}$ ), 153.9 (s, $\left.C \mathrm{H}_{\mathrm{Py}}\right), 153.0$ (s, $\left.C_{\mathrm{Py}}\right), 140.6$ (s, $C \mathrm{H}_{\mathrm{Py}}$ ), $135.4\left(\mathrm{~d}, J_{\mathrm{PC}}=13.2 \mathrm{~Hz}, C_{i p s o} \mathrm{Ph}\right), 135.0\left(\mathrm{~d}, J_{\mathrm{PC}}=14.0 \mathrm{~Hz}, C_{i p s o} \mathrm{Ph}\right), 134.1$ $\left(\mathrm{d}, J_{\mathrm{PC}}=19.9 \mathrm{~Hz}, C \mathrm{H}_{\mathrm{Ph}}\right), 132.9\left(\mathrm{~d}, J_{\mathrm{PC}}=18.8 \mathrm{~Hz}, C \mathrm{H}_{\mathrm{Ph}}\right), 130.5(\mathrm{~s}$, $\left.C \mathrm{H}_{\mathrm{Ph}}\right), 129.9\left(\mathrm{~s}, C \mathrm{H}_{\mathrm{Ph}}\right), 129.5\left(\mathrm{~d}, J_{\mathrm{PC}}=7.3 \mathrm{~Hz}, C \mathrm{H}_{\mathrm{Ph}}\right), 129.3\left(\mathrm{~d}, J_{\mathrm{PC}}=\right.$ $\left.6.5 \mathrm{~Hz}, C \mathrm{H}_{\mathrm{Ph}}\right), 125.1\left(\mathrm{~d}, J_{\mathrm{PC}}=5.8 \mathrm{~Hz}, C \mathrm{H}_{\mathrm{Im}-4,5)}\right) 122.8\left(\mathrm{~s}, C \mathrm{H}_{\mathrm{Im}-4,5)}\right.$, 115.9 (s, $C \mathrm{HPy}_{\mathrm{Py}}$ ), 111.7 (s, CHРy), 51.9 (d, JPC $=17.5 \mathrm{~Hz}, \mathrm{PCH}_{2}$ ); IR (THF): v $v_{\mathrm{CO}} 2016$ (s), 1930 (s), 1907 (s) $\mathrm{cm}^{-1}$; Anal. Found: C, 51.28; $\mathrm{H}, 2.71$; N, 7.46. Calcd. (\%) for $\mathrm{C}_{24} \mathrm{H}_{18} \mathrm{BrMnN}_{3} \mathrm{O}_{3} \mathrm{P}: \mathrm{C}, 51.27$; $\mathrm{H}, 3.23$; N, 7.47.

Synthesis of PCN Tridentate Complex [15](OTf). To a solution of the mixture of complexes $\mathbf{1 3}$ and $\mathbf{1 4}(0.20 \mathrm{~g}, 0.36 \mathrm{mmol})$ in $\mathrm{CH}_{2} \mathrm{Cl}_{2}$ $(20 \mathrm{~mL}) \operatorname{AgOTf}(0.10 \mathrm{~g}, 0.39 \mathrm{mmol})$ was added as a solid. The reaction mixture was stirred at RT for $2 \mathrm{~h}$. After filtration through Celite and evaporation of the solvent under vacuum, the crude residue was crystallized in $\mathrm{THF} / \mathrm{Et}_{2} \mathrm{O}$ mixture affording complex [15](OTf) as a greenyellow powder $(0.17 \mathrm{~g}, 76 \%)$. Single crystals suitable for XRD were obtained by slow evaporation of the complex solution in $\mathrm{CH}_{2} \mathrm{Cl}_{2}$ at $\mathrm{RT}$. ${ }^{1} \mathrm{H}$ NMR $\left(400 \mathrm{MHz}, \mathrm{CDCl}_{3}, 25^{\circ} \mathrm{C}\right): \delta 8.58$ (brs, $\left.2 \mathrm{H}, \mathrm{CH}_{\mathrm{Ar}}\right), 8.25$ (brs, $\left.1 \mathrm{H}, \mathrm{C} H_{\mathrm{Ar}}\right), 8.10\left(\mathrm{brs}, 2 \mathrm{H} \mathrm{C} H_{\mathrm{Ar}}\right), 7.46-7.44\left(\mathrm{~m}, 11 \mathrm{H}, \mathrm{C} H_{\mathrm{Ar}}\right), 5.51$ (brs, $\left.2 \mathrm{H}, \mathrm{PCH} \mathrm{H}_{2}\right) ;{ }^{31} \mathrm{P}\left\{{ }^{1} \mathrm{H}\right\} \mathrm{NMR}\left(162 \mathrm{MHz}, \mathrm{CD}_{2} \mathrm{Cl}_{2}, 25^{\circ} \mathrm{C}\right): \delta 97.4(\mathrm{~s})$; ${ }^{13} \mathrm{C}\left\{{ }^{1} \mathrm{H}\right\}$ NMR $\left(101 \mathrm{MHz}, \mathrm{CDCl}_{3}, 25^{\circ} \mathrm{C}\right): \delta 218.4\left(\mathrm{~d}, J_{\mathrm{PC}}=9.7 \mathrm{~Hz}, C \mathrm{O}\right)$, $212.7\left(\mathrm{~d}, J_{\mathrm{PC}}=17.7 \mathrm{~Hz}, C \mathrm{O}\right), 206.5\left(\mathrm{~d}, J_{\mathrm{PC}}=18.9 \mathrm{~Hz}, C \mathrm{~N}_{2}\right), 156.0(\mathrm{~s}$, $\left.C \mathrm{H}_{\mathrm{Py}}\right), 154.6\left(\mathrm{~s}, C_{\mathrm{Py}}\right), 141.8\left(\mathrm{~s}, C \mathrm{H}_{\mathrm{Ar}}\right), 133.8\left(\mathrm{~d}, J_{\mathrm{PC}}=42.8 \mathrm{~Hz}, C_{i p s o \mathrm{Ph}}\right)$, $131.6\left(\mathrm{~d}, J_{\mathrm{PC}}=2.4 \mathrm{~Hz}, C \mathrm{HPh}_{\mathrm{Ph}}\right), 131.4\left(\mathrm{~d}, J_{\mathrm{PC}}=11.0 \mathrm{~Hz}, C \mathrm{HPh}_{\mathrm{Ph}}, 129.5(\mathrm{~d}\right.$, $\left.J_{\mathrm{PC}}=10.4 \mathrm{~Hz}, C \mathrm{H}_{\mathrm{Ph}}\right), 125.4\left(\mathrm{~d}, J_{\mathrm{PC}}=6.0 \mathrm{~Hz}, C \mathrm{H}_{\mathrm{Im}-4,5}\right), 123.4\left(\mathrm{~s}, C \mathrm{H}_{\mathrm{Im}-}\right.$ 4,5), 120.8 (s, $\left.C \mathrm{HPy}_{\mathrm{Py}}\right), 114.2$ (s, $\left.C \mathrm{HPy}_{\mathrm{Py}}\right), 58.1$ (d, $\left.J_{\mathrm{PC}}=37.4 \mathrm{~Hz}, \mathrm{PCH}_{2}\right)$; IR (THF): $v_{\mathrm{CO}} 2052$ (w), 1969 (vs), 1941 (s) $\mathrm{cm}^{-1}$; Anal. Found: C, 47.60; $\mathrm{H}, 2.45 ; \mathrm{N}, 6.45$. Calcd. (\%) for $\mathrm{C}_{25} \mathrm{H}_{18} \mathrm{~F}_{3} \mathrm{MnN}_{3} \mathrm{O}_{6} \mathrm{PS}: \mathrm{C}, 47.56 ; \mathrm{H}, 2.87$; $\mathrm{N}, 6.66$; $\mathrm{MS}\left(\mathrm{ES}^{+}\right): \mathrm{m} / z$ : $482.0[\mathrm{M}]^{+}$; HRMS $\left(\mathrm{ES}^{+}\right)$: calcd. for $\mathrm{C}_{24} \mathrm{H}_{18} \mathrm{MnN}_{3} \mathrm{O}_{3} \mathrm{P}^{+}$482.0466; found, 482.0466 ( $\varepsilon_{\mathrm{r}}=0 \mathrm{ppm}$ ).

Synthesis of PCN Bidentate Complex 16. To a stirred suspension of $[\mathbf{1 0}](\mathrm{OTs})(0.32 \mathrm{~g}, 0.60 \mathrm{mmol})$ in THF $(20 \mathrm{~mL})$, a solution of KHMDS (0.5 M in toluene, $1.3 \mathrm{~mL}, 0.66 \mathrm{mmol}$ ) was added at RT and then the resulting brown suspension was sonicated during $5 \mathrm{~min}$ and stirred for additional $15 \mathrm{~min}$. Solid $\left[\mathrm{Mn}(\mathrm{CO})_{5} \mathrm{Br}\right](0.17 \mathrm{~g}, 0.60 \mathrm{mmol})$ was added in one portion inducing a vigorous $\mathrm{CO}$ evolution. The resulting dark orange solution was then stirred for $2 \mathrm{~h}$ at $50{ }^{\circ} \mathrm{C}$. After cooling the reaction mixture to RT, filtration through Celite and evaporation of the solvent under vacuum, the crude product was purified by column chromatography on basic activated aluminium oxide using THF/toluene mixture as eluent affording complex $\mathbf{1 6}$ as a yellow-orange powder $(0.21 \mathrm{~g}, 60 \%)$. Single crystals suitable for XRD were obtained by slow evaporation of complex solution in $\mathrm{CH}_{2} \mathrm{Cl}_{2}$ /toluene mixture at RT. ${ }^{1} \mathrm{H}$ NMR $\left(400 \mathrm{MHz}, \mathrm{CD}_{2} \mathrm{Cl}_{2}, 25^{\circ} \mathrm{C}\right): \delta 8.57(\mathrm{~d}, J=4.7 \mathrm{~Hz}$, $\left.1 \mathrm{H}, \mathrm{C} H_{\mathrm{Ar}}\right), 8.00-7.91\left(\mathrm{~m}, 2 \mathrm{H}, \mathrm{CH}_{\mathrm{Ar}}\right), 7.68-7.65\left(\mathrm{~m}, 1 \mathrm{H}, \mathrm{CH}_{\mathrm{Ar}}\right), 7.57-$ $7.50\left(\mathrm{~m}, 3 \mathrm{H}, \mathrm{C} H_{\mathrm{Ar}}\right), 7.43-7.32\left(\mathrm{~m}, 4 \mathrm{H}, \mathrm{C} H_{\mathrm{Ar}}\right), 7.27-7.13(\mathrm{~m}, 5 \mathrm{H}$, 
$\left.\mathrm{C}_{\mathrm{Ar}}\right), 5.74\left(\mathrm{~d}, J_{\mathrm{HH}}=16.0 \mathrm{~Hz}, 1 \mathrm{H}, \mathrm{PyCH}_{2}\right), 5.64\left(\mathrm{~d}, J_{\mathrm{HH}}=16.0 \mathrm{~Hz}, 1 \mathrm{H}\right.$, $\left.\mathrm{PyCH}_{2}\right), 4.96-5.05\left(\mathrm{~m}, 2 \mathrm{H}, \mathrm{PCH}{ }_{2}\right) ;{ }^{31} \mathrm{P}\left\{{ }^{1} \mathrm{H}\right\}$ NMR $\left(162 \mathrm{MHz}, \mathrm{CDCl}_{3}\right.$, $\left.25{ }^{\circ} \mathrm{C}\right): \delta 71.2(\mathrm{~s}) ;{ }^{13} \mathrm{C}\left\{{ }^{1} \mathrm{H}\right\} \mathrm{NMR}\left(101 \mathrm{MHz}, \mathrm{CD}_{2} \mathrm{Cl}_{2}, 25{ }^{\circ} \mathrm{C}\right): \delta 222.8$ (brd, $\left.J_{\mathrm{PC}}=16.8 \mathrm{~Hz}, C \mathrm{C}\right), 220.8\left(\mathrm{brd}, J_{\mathrm{PC}}=30.1 \mathrm{~Hz}, C O\right), 220.2$ (brd, $\left.J_{\mathrm{PC}}=20.8 \mathrm{~Hz}, C \mathrm{O}\right), 197.7\left(\mathrm{~d}, J_{\mathrm{PC}}=17.5 \mathrm{~Hz}, C_{2}\right), 156.5\left(\mathrm{~s}, C_{\mathrm{Py}}\right), 150.0$ (s, $\left.C \mathrm{H}_{\mathrm{Ar}}\right), 137.6\left(\mathrm{~s}, C \mathrm{H}_{\mathrm{Ar}}\right), 135.7\left(\mathrm{~d}, J_{\mathrm{PC}}=36.6 \mathrm{~Hz}, C_{i p s o} \mathrm{Ph}\right), 134.9(\mathrm{~d}$, $\left.J_{\mathrm{PC}}=9.8 \mathrm{~Hz}, C \mathrm{H}_{\mathrm{Ar}}\right), 132.0\left(\mathrm{~s}, C \mathrm{H}_{\mathrm{Ar}}\right), 130.7\left(\mathrm{~m}, 2 C \mathrm{H}_{\mathrm{Ar}}\right), 129.4\left(\mathrm{~d}, J_{\mathrm{PC}}\right.$ $\left.=9.0 \mathrm{~Hz}, C \mathrm{H}_{\mathrm{Ar}}\right), 129.3\left(\mathrm{~d}, J_{\mathrm{PC}}=9.0 \mathrm{~Hz}, C \mathrm{H}_{\mathrm{Ar}}\right), 125.2\left(\mathrm{~s}, C \mathrm{H}_{\mathrm{Ar}}\right), 123.4$

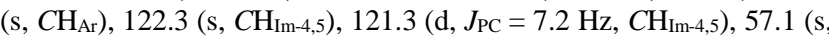
$\mathrm{PyCH}_{2}$ ), 52.3 (d, $J_{\mathrm{PC}}=31.5 \mathrm{~Hz}, \mathrm{PCH}_{2}$ ); IR (THF): $v_{\mathrm{CO}} 2018$ (s), 1941 (s), 1913 (s) $\mathrm{cm}^{-1}$; Anal. Found: C, 51.61; H, 3.38; N, 7.03. Calcd. (\%) for $\mathrm{C}_{25} \mathrm{H}_{20} \mathrm{BrMnN}_{3} \mathrm{O} 3 \mathrm{P}: \mathrm{C}, 52.11 ; \mathrm{H}, 3.50 ; \mathrm{N}, 7.29 ; \mathrm{MS}\left(\mathrm{ES}^{+}\right): \mathrm{m} / \mathrm{z}$ $576.0[\mathrm{M}+\mathrm{H}]^{+}$; HRMS $\left(\mathrm{ES}^{+}\right)$: calcd. for $\mathrm{C}_{25} \mathrm{H}_{21} \mathrm{BrMnN}_{3} \mathrm{O}_{3} \mathrm{P}$ 575.9884; found, $575.9893\left(\varepsilon_{\mathrm{r}}=1.6 \mathrm{ppm}\right)$.

Synthesis of PCN Tridentate Complex [17](OTf). To a solution of $16(0.20 \mathrm{~g}, 0.34 \mathrm{mmol})$ in $\mathrm{CH}_{2} \mathrm{Cl}_{2}(20 \mathrm{~mL})$ AgOTf $(0.10 \mathrm{~g}, 0.37$ $\mathrm{mmol}$ ) was added as a solid. The reaction mixture was stirred at RT for $2 \mathrm{~h}$. After filtration through Celite and evaporation of the solvent under vacuum, [17](OTf) was obtained as a yellow-brown powder $(0.21 \mathrm{~g}$, $96 \%$ ). Single crystals suitable for XRD were obtained by slow evaporation of the concentrated $\mathrm{CH}_{2} \mathrm{Cl}_{2}$ solution at RT. ${ }^{1} \mathrm{H} \mathrm{NMR}(400 \mathrm{MHz}$, $\mathrm{CD}_{2} \mathrm{Cl}_{2}, 25^{\circ} \mathrm{C}$ ): $\delta 8.02\left(\mathrm{dd}, J=7.4\right.$ and $\left.10.9 \mathrm{~Hz}, 2 \mathrm{H}, \mathrm{CH} H_{\mathrm{Ar}}\right), 7.88$ (s, $\left.1 \mathrm{H}, \mathrm{CH}_{\mathrm{Ar}}\right), 7.82-7.70\left(\mathrm{~m}, 5 \mathrm{H}, \mathrm{CH}_{\mathrm{Ar}}\right), 7.66-7.61\left(\mathrm{~m}, 2 \mathrm{H}, \mathrm{C} H_{\mathrm{Ar}}\right), 7.16(\mathrm{t}$, $\left.J=7.5 \mathrm{~Hz}, 1 \mathrm{H}, \mathrm{CH}_{\mathrm{Ar}}\right), 6.96\left(\mathrm{t}, J=7.8 \mathrm{~Hz}, 2 \mathrm{H}, \mathrm{C} H_{\mathrm{Ar}}\right), 6.60(\mathrm{t}, J=6.6$ $\left.\mathrm{Hz}, 1 \mathrm{H}, \mathrm{C} H_{\mathrm{Ar}}\right), 5.95\left(\mathrm{t}, J=8.0 \mathrm{~Hz}, 2 \mathrm{H}, \mathrm{CH}_{\mathrm{Ar}}\right), 5.89(\mathrm{~d}, J=16.0 \mathrm{~Hz}, 1 \mathrm{H}$, $\left.\mathrm{PyCH}_{2}\right), 5.54\left(\mathrm{~d}, J=16.0 \mathrm{~Hz}, 1 \mathrm{H}, \mathrm{PyCH}_{2}\right), 5.36(\mathrm{t}, J=12.0 \mathrm{~Hz}, 1 \mathrm{H}$, $\left.\mathrm{PCH}_{2}\right), 4.91\left(\mathrm{dd}, J=8.9\right.$ and $\left.12.0 \mathrm{~Hz}, 1 \mathrm{H}, \mathrm{PCH}_{2}\right) ;{ }^{31} \mathrm{P}\left\{{ }^{1} \mathrm{H}\right\} \mathrm{NMR}(162$ $\left.\mathrm{MHz}, \mathrm{CD}_{2} \mathrm{Cl}_{2}, 25{ }^{\circ} \mathrm{C}\right): \delta 93.3(\mathrm{~s}) ;{ }^{13} \mathrm{C}\left\{{ }^{1} \mathrm{H}\right\}$ NMR $\left(101 \mathrm{MHz}, \mathrm{CD}_{2} \mathrm{Cl}_{2}\right.$, $25{ }^{\circ} \mathrm{C}$ ): $\delta 219.6\left(\mathrm{brd}, J_{\mathrm{PC}}=25.5 \mathrm{~Hz}, C O\right), 218.7$ (brd, $J_{\mathrm{PC}}=18.5 \mathrm{~Hz}$, $C O), 216.9\left(\right.$ brd, $\left.J_{\mathrm{PC}}=23.5 \mathrm{~Hz}, C \mathrm{O}\right), 202.9\left(\mathrm{~d}, J_{\mathrm{PC}}=22.3 \mathrm{~Hz}, C \mathrm{~N}_{2}\right)$, $157.7\left(\mathrm{~d}, J_{\mathrm{PC}}=3.4 \mathrm{~Hz}, C \mathrm{H}_{\mathrm{Ar}}\right), 156.9\left(\mathrm{~s}, C_{\mathrm{Py}}\right), 139.5\left(\mathrm{~s}, C \mathrm{H}_{\mathrm{Ar}}\right), 134.4(\mathrm{~d}$, $\left.J_{\mathrm{PC}}=10.7 \mathrm{~Hz}, C \mathrm{H}_{\mathrm{Ar}}\right), 133.6\left(\mathrm{~d}, J_{\mathrm{PC}}=2.0 \mathrm{~Hz}, C \mathrm{H}_{\mathrm{Ar}}\right), 131.2\left(\mathrm{~d}, J_{\mathrm{PC}}=2.4\right.$ $\left.\mathrm{Hz}, C \mathrm{H}_{\mathrm{Ar}}\right), 130.4\left(\mathrm{~d}, J_{\mathrm{PC}}=9.9 \mathrm{~Hz}, C \mathrm{H}_{\mathrm{Ar}}\right), 129.7\left(\mathrm{~d}, J_{\mathrm{PC}}=9.7 \mathrm{~Hz}, C \mathrm{H}_{\mathrm{Ar}}\right)$, $129.6\left(\mathrm{~d}, J_{\mathrm{PC}}=9.4 \mathrm{~Hz}, C \mathrm{H}_{\mathrm{Ar}}\right), 127.5\left(\mathrm{~d}, J_{\mathrm{PC}}=36.6 \mathrm{~Hz}, C_{i p s o} \mathrm{Ph}\right), 127.4$ $\left(\mathrm{s}, C \mathrm{H}_{\mathrm{Ar}}\right), 126.3\left(\mathrm{~s}, C \mathrm{H}_{\mathrm{Ar}}\right), 124.7\left(\mathrm{~s}, C \mathrm{H}_{\mathrm{Ar}}\right), 123.1\left(\mathrm{~d}, J_{\mathrm{PC}}=1.7 \mathrm{~Hz}\right.$, $\left.\mathrm{CH}_{\mathrm{Ar}}\right), 55.8\left(\mathrm{~d}, \mathrm{~J}_{\mathrm{PC}}=37.9 \mathrm{~Hz}, \mathrm{PCH}_{2}\right), 55.6\left(\mathrm{~s}, \mathrm{PyCH}_{2}\right)$; IR (THF): $v_{\mathrm{CO}}$ 2024 (s), 1947 (s), 1929 (s) cm ${ }^{-1}$; Anal. Found: C, 47.17; H, 2.84; N, 6.34. Calcd. (\%) for $\mathrm{C}_{26} \mathrm{H}_{20} \mathrm{~F}_{3} \mathrm{MnN}_{3} \mathrm{O}_{6} \mathrm{PS}$. $0.25\left(\mathrm{CH}_{2} \mathrm{Cl}_{2}\right)$ : C, 47.29; $\mathrm{H}$, 3.10; N, 6.30; MS $\left(\mathrm{ES}^{+}\right): m / z: 496.1[\mathrm{M}]^{+}$; HRMS $\left(\mathrm{ES}^{+}\right)$: calcd. for $\mathrm{C}_{25} \mathrm{H}_{20} \mathrm{MnN}_{3} \mathrm{O}_{3} \mathrm{P}$ 496.0623; found, $496.0626\left(\varepsilon_{\mathrm{r}}=0.6 \mathrm{ppm}\right)$.

General procedure for the hydrogenation of acetophenone. Under an argon atmosphere, a Schlenk tube was charged with selected complex and acetophenone $(1 \mathrm{mmol}, 117 \mu \mathrm{L})$ in $2 \mathrm{~mL}$ of $t-\mathrm{AmOH}$, the solution was placed in a steel autoclave. Then $t$-BuOK $(10 \mathrm{~mol} \%)$ was added and the autoclave was immediately pressurized with $\mathrm{H}_{2}(50 \mathrm{bar})$ and kept under stirring in an oil bath at 60 or $100{ }^{\circ} \mathrm{C}$ for 24 hours. After cooling the reactor to room temperature and release of $\mathrm{H}_{2}$, the reaction mixture was diluted with ethyl acetate $(2.0 \mathrm{~mL})$ and filtered through a small pad of Celite ( $2 \mathrm{~cm}$ in a Pasteur pipette) and the solution was analyzed by $\mathrm{GC}$ and ${ }^{1} \mathrm{H}$ NMR spectroscopy. To isolate the product, the crude residue was purified by column chromatography on silica using petroleum ether/ethyl acetate mixtures as eluent.

Single-crystal X-ray diffraction analyses. Single crystals suitable for X-ray diffraction were coated with paratone oil and mounted onto the goniometer. The X-ray crystallographic data were obtained at low temperature from a Bruker Apex2 diffractometer (MoKa radiation source) equipped with an Oxford Cryosystem. The structures have been solved with Superflip ${ }^{32}$ and refined by means of least-square procedures on $\mathrm{F}$ or $\mathrm{F}^{2}$ using the PC version of the program CRYSTALS. ${ }^{33}$ The scattering factors for all the atoms were used as listed in the International Tables for X-ray Crystallography. Absorption correction was performed using a multi-scan procedure. All non-hydrogen atoms were refined anisotropically. The $\mathrm{H}$ atoms were located in a difference map, but those attached to carbon atoms were repositioned geometrically. The $\mathrm{H}$ atoms were initially refined with soft restraints on the bond lengths and angles to regularise their geometry and $\mathrm{U}_{[\text {[iso] }}(\mathrm{H}$ ) (in the range 1.2-1.5 times $U_{[\text {eq] }}$ of the parent atom), after which the positions were refined with riding constraints. Detailed crystallographic data and structural refinement parameters are given in Table S1 of the Supporting Information.

\section{ASSOCIATED CONTENT}

\section{Supporting Information}

The Supporting Information is available free of charge on the ACS Publications website.

Copies of ${ }^{1} \mathrm{H},{ }^{31} \mathrm{P}$ and ${ }^{13} \mathrm{C}$ NMR spectra, representation of $\mathrm{X}$-ray structure of $[4](\mathrm{OTs})$, crystallographic data and structural refinement parameters for all studied compounds (PDF).

\section{Accession Codes}

CCDC 2042375 [4](OTs), CCDC 2042377 13, CCDC 2042380 14, CCDC 2042376 16, CCDC 2042403 [12](OTf), CCDC 2042379 [15](OTf), and CCDC 2042378 [17](OTf) contain the supplementary crystallographic data for this paper. These data can be obtained free of charge via www.ccdc.cam.ac.uk/data_request/cif, or by emailing data_request@ccdc.cam.ac.uk, or by contacting The Cambridge Crystallographic Data Centre, 12 Union Road, Cambridge CB2 1EZ, UK; fax: +44 1223336033.

\section{AUTHOR INFORMATION}

\section{Corresponding Authors}

*E-mails: yves.canac@1cc-toulouse.fr, jean-baptiste.sortais@1cctoulouse.fr, dmitry.valyaev@1cc-toulouse.fr

Yves Canac: 0000-0002-3747-554X

Jean-Baptiste Sortais: 0000-0003-1178-8588

Dmitry A. Valyaev: 0000-0002-1772-844X

\section{Notes}

The authors declare no competing financial interests.

\section{ACKNOWLEDGMENT}

The authors thank the Centre National de la Recherche Scientifique (CNRS), the Université Toulouse III, Paul Sabatier, and the Institut Universitaire de France (IUF) for general support of this project. R.B. is grateful to the Embassy of Yemen in Paris, and the program Pause for financial support.

\section{REFERENCES}

(1) (a) Bourissou, D.; Guerret, O.; Gabbaï, F. P.; Bertrand, G. Stable carbenes. Chem. Rev. 2000, 100, 39-91. (b) Y. Canac, M. Soleilhavoup, S. Conejero, G. Bertrand, Stable non-N-heterocyclic carbenes (non-NHC): recent progress. J. Organomet. Chem. 2004, 689, 38573865. (c) Melaimi, M.; Soleilhavoup, M.; G. Bertrand, G. Stable carbenes and related species beyond diaminocarbenes. Angew. Chem. Int. Ed. 2010, 49, 8810-8849. (d) Hopkinson, M. N.; Richter, C.; Schedler, M.; Glorius, F. An overview of N-Heterocyclic carbenes. Nature 2014, 510, 485-496. (e) Vivancos, A.; Segarra, C.; Albrecht, M. Mesoionic and related less heteroatom-stabilized N-heterocyclic carbene complexes: synthesis, catalysis, and other applications. Chem. Rev. 2018, 118, 9493-9586. (f) Sau, S. C.; Hota, P. K.; Mandal, S. K.; Soleilhavoup, M.; Bertrand, G. Stable abnormal N-heterocyclic carbenes and their applications. Chem. Soc. Rev. 2020, 49, 1233-1252.

(2) (a) Froese, R. D. J.; Lombardi, C.; Pompeo, M.; Rucker, R. P.; Organ, M. G. Designing Pd-N-heterocyclic carbene complexes for high reactivity and selectivity for cross-coupling applications. Acc. Chem. Res. 2017, 50, 2244-2253. (b) Janssen-Muller, D.; Schlepphorst, C.; Glorius, F. Privileged chiral N-heterocyclic carbene ligands for asymmetric transition-metal catalysis. Chem. Soc. Rev. 2017, 46 , 4845-4854. (c) Melaimi, M.; Jazzar, R.; Soleilhavoup, M.; Bertrand, G. Cyclic (alkyl)(amino)carbenes (CAACs): recent developments. Angew. Chem. Int. Ed. 2017, 56, 10046-10068. (d) Ogba, O. M.; Warner, N. C.; O'Leary, D. J.; Grubbs, R. H. Recent advances in rutheniumbased olefin metathesis. Chem. Soc. Rev. 2018, 47, 4510-4544.

(3) (a) Mata, J. A.; Poyatos, M.; Peris, E. Structural and catalytic properties of chelating bis- and tris-N-heterocyclic carbenes. Coord Chem. Rev. 2007, 251, 841-859. (b) Poyatos, M.; Mata, J. A.; Peris, E. 
Complexes with poly(N-heterocyclic carbene) ligands: structural features and catalytic applications. Chem. Rev. 2009, 109, 3677-3707. (c) Schick, S.; Pape, T.; Hahn, F. E. Coordination chemistry of bidentate bis(NHC) ligands with two different NHC donors. Organometallics 2014, 33, 4035-4041. (d) Charra, V.; de Frémont, P.; Braunstein, P. Multidentate N-heterocyclic carbene complexes of the $3 \mathrm{~d}$ metals: synthesis, structure, reactivity and catalysis. Coord. Chem. Rev. 2017, 341 , 53-176.

(4) (a) Chen, J. C. C.; Lin, I. J. B. Palladium complexes containing a hemilabile pyridylcarbene ligand. Organometallics 2000, 19, 51135121. (b) Tulloch, A. A. D.; Danopoulos, A. A.; Kleinhenz, S.; Light, M. E.; Hursthouse, M. B.; Eastham, G. Structural diversity in pyridine$\mathrm{N}$-functionalized carbene copper(I) complexes. Organometallics 2001 , 20, 2027-2031. (c) Magill, A. M.; McGuinness, D. S.; Cavell, K. J.; Britovsek, G. J. P.; Gibson, V. C.; White, A. J. P.; Williams, D. J.; White, A. H.; Skelton, B. W. Palladium(II) complexes containing mono-, bi- and tridentate carbene ligands. Synthesis, characterization and application as catalysts in $\mathrm{C}-\mathrm{C}$ coupling reactions. J. Organomet. Chem. 2001, 617-618, 546-560. (d) Valyaev, D. A.; Willot, J.; Mangin, L. P.; Zargarian, D.; Lugan, N. Manganese-mediated synthesis of an $\mathrm{NHC}$ core non-symmetric pincer ligand and evaluation of its coordinating propeties. Dalton Trans. 2017, 46, 10193-10196. (e) Jiang, Y.; Gendy, C.; Roesler, R. Nickel, ruthenium, and rhodium NCN-pincer complexes featuring a six-membered $\mathrm{N}$-heterocyclic carbene central moiety and pyridyl pendant arms. Organometallics 2018, 37, $1123-1132$

(5) (a) Hahn, F. E.; Jahnke, M. C.; Pape, T. Synthesis of palladium and platinum complexes with phosphine-functionalized benzimidazolin-2-ylidene ligands. Organometallics 2006, 25, 5927-5936. (b) Danopoulos, A. A.; Tsoureas, N.; Macgregor, S. A.; Smith, C. Phosphineand pyridine-functionalized $\mathrm{N}$-heterocyclic carbene methyl and allyl complexes of palladium. Unexpected regiospecificity of the protonation reaction of the dimethyl complexes. Organometallics 2007, 26, 253-263. (c) Hill, A. F.; McQueen, C. M. A. Dihydroperimidine-derived $\mathrm{N}$-heterocyclic pincer carbene complexes via double $\mathrm{C}-\mathrm{H}$ activation. Organometallics 2012, 31, 8051-8054. (d) Salem, H.; Schmitt, M.; Herrlich, U.; Kühnel, E.; Brill, M.; Nägele, P.; Bogado, A. L.; Rominger, F.; Hofmann, P. Bulky N-phosphinomethyl-functionalized $\mathrm{N}$-heterocyclic carbene chelate ligands: synthesis, molecular geometry, electronic structure, and their ruthenium alkylidene complexes. Organometallics 2013, 32, 29-46; (e) Fuku-en, S.; Yamamoto, J.; Kojima, S.; Yamamoto Y. Synthesis and application of new dipyrido-annulated Nheterocyclic carbene with phosphorus substituents. Chem. Lett. 2014 43, 468-470. (f) Valyaev, D. A.; Filippov, O. A.; Lugan, N.; Lavigne, G.; Ustynyuk, N. A. Umpolung of methylenephosphonium ions in their manganese half-sandwich and application to the synthesis of chiral phosphorus-containing ligand scaffolds. Angew. Chem. Int. Ed. 2015 , 54, 6315-6319. (g) Plikhta, A.; Pöthig, A.; Herdtweck, E.; Rieger, B. Toward new organometallic architectures: synthesis of carbene-centered rhodium and palladium bisphosphine complexes. Stability and reactivity of $\left[\mathrm{PC}^{\mathrm{BIm}} \mathrm{PRh}(\mathrm{L})\right]\left[\mathrm{PF}_{6}\right]$ pincers. Inorg. Chem. 2015, 54, $9517-$ 9528. (h) Matoba, K.; Eizawa, A.; Nishimura, S.; Arashiba, K.; Nakajima, K.; Nishibayashi, Y. Practical synthesis of a PCP-type pincer ligand and its metal complexes. Synthesis 2018, 50, 1015-1019.

(6) (a) Aihara, H.; Matsuo, T.; Kawaguchi, H. Titanium N-heterocyclic carbene complexes incorporating an imidazolium-linked bis(phenol). Chem. Commun. 2003, 2204-2205. (b) Waltman, A. W.; Grubbs, R. H. A new class of chelating N-Heterocyclic carbene ligands and their complexes with palladium. Organometallics 2004, 23, 3105-3107. (c) Romain, C.; Brelot, L.; Bellemin-Laponnaz, S.; Dagorne, S. Synthesis and structural characterization of a novel family of titanium complexes bearing a tridentate bis-phenolate-N-heterocyclic carbene dianionic ligand and their use in the controlled ROP of raclactide. Organometallics 2010, 29, 1191-1198. (d) Taakili, R.; Canac, Y. NHC core pincer ligands exhibiting two anionic coordinating extremities. Molecules 2020, 25, 2231.

(7) (a) Benaissa, I.; Taakili, R.; Lugan, N.; Canac, Y. A convenient access to N-phosphonio-substituted NHC metal complexes $[\mathrm{M}=\mathrm{Ag}(\mathrm{I})$, Rh(I), Pd(II)]. Dalton Trans. 2017, 46, 12293-12305. (b) Barthes, C.; Bijani, C.; Lugan, N.; Canac, Y. A palladium(II) complex of a $\mathrm{C}_{4}$ chelating bis(NHC) diphosphonium bis(ylide) ligand. Organometallics
2018, 37, 673-678. (c) Taakili, R.; Lepetit, C.; Duhayon, C.; Valyaev, D. A.; Lugan, N.; Canac, Y. Palladium(II) pincer complexes of a C,C,C-NHC, diphosphonium bis(ylide) ligand. Dalton Trans. 2019, 48, 1709-1721. (d) Taakili, R.; Barthes, C.; Goëffon, A.; Lepetit, C.; Duhayon, C.; Valyaev, D. A.; Canac, Y. NHC core phosphonium ylidebased palladium(II) pincer complexes: the second ylide extremity makes the difference. Inorg. Chem. 2020, 59, 7082-7096.

(8) (a) Valyaev, D. A.; Lavigne, G.; Lugan, N. Manganese organometallic compounds in homogeneous catalysis: past, present and prospects. Coord. Chem. Rev. 2016, 308, 191-235. (b) Trovitch, R. J. The emergence of manganese-based carbonyl hydrosilylation catalysts. Acc. Chem. Res. 2017, 50, 2842-2852. (c) Hu, Y.; Zhou, B.; Wang, C. Inert $\mathrm{C}-\mathrm{H}$ bond transformations enabled by organometallic manganese catalysis. Acc. Chem. Res. 2018, 51, 816-827. (d) Stanbury, M.; Compain, J.-D.; Chardon-Noblat, S. Electro and photoreduction of $\mathrm{CO}_{2}$ driven by manganese-carbonyl molecular catalysts. Coord. Chem. Rev. 2018, 361, 120-137. (e) Kaim, V.; Kaur-Ghumaan, S. Manganese complexes: hydrogen generation and oxidation. Eur. J. Inorg. Chem. 2019, 5041-5051.

(9) (a) Garbe, M.; Junge, K.; Beller, M. Homogeneous catalysis by manganese-based pincer complexes. Eur. J. Org. Chem. 2017, 43444362. (b) Maji, B.; Barman, M. K. Recent developments of manganese complexes for catalytic hydrogenation and dehydrogenation reactions. Synthesis 2017, 49, 3377-3393. (c) Kallmeier, F.; Kempe, R. Manganese complexes for (de)hydrogenation catalysis: a comparison to cobalt and iron catalysts. Angew. Chem. Int. Ed. 2018, 57, 46-60. (d) Manganese-catalyzed hydrogenation and hydrogen transfer reactions. Sortais, J.-B.; Buhaibeh, R.; Canac, Y. Manganese Catalysis in Organic Synthesis, Ed. Sortais, J.-B. Wiley-VCH, in press.

(10) (a) Elangovan, S., Topf, C., Fischer, S., Jiao, H.; Spannenberg, A.; Baumann, W.; Ludwig, R.; Junge, K.; Beller, M. Selective catalytic hydrogenations of nitriles, ketones, and aldehydes by well-defined manganese pincer complexes. J. Am. Chem. Soc. 2016, 138, 88098814. (b) Kallmeier, F., Irrgang, T., Dietel, T., Kempe, R. Highly active and selective manganese $\mathrm{C}=\mathrm{O}$ bond hydrogenation catalysts: the importance of the multidentate ligand, the ancillary ligands, and the oxidation state. Angew. Chem. Int. Ed. 2016, 55, 11806-11809. (c) Bruneau-Voisine, A.; Wang, D.; Roisnel, T.; Darcel, C.; Sortais, J.-B. Hydrogenation of ketones with a manganese $\mathrm{PN}^{3} \mathrm{P}$ pincer pre-catalyst. Catal. Commun. 2017, 92, 1-4.

(11) (a) Espinosa-Jalapa, N. A., Nerush, A., Shimon, L. J. W., Leitus, G.; Avram, L.; Ben-David, Y.; Milstein, D. Manganesecatalyzed hydrogenation of esters to alcohols. Chem. Eur. J. 2017, 23, 5934-5938. (b) Zhang, L.; Tang, Y.; Han, Z.; Ding, K. Lutidine-based chiral pincer manganese catalysts for enantioselective hydrogenation of ketones. Angew. Chem. Int. Ed. 2019, 58, 4973-4977.

(12) Perez, M.; Elangovan, S.; Spannenberg, A.; Junge, K.; Beller, M. Molecularly defined manganese pincer complexes for selective transfer hydrogenation of ketones. ChemSusChem 2017, 10, 83-86.

(13) Yang, W.; Chernyshov, I. Y.; van Schendel, R. K. A.; Weber, M.; Müller, C.; Filonenko, G. A.; Pidko, E. A. Phosphine ligand hemilability as a route towards robust and efficient hydrogenation with $\mathrm{Mn}(\mathrm{I})$ complexes. ChemRxiv 2020, 1-7.

(14) (a) van Putten, R.; Uslamin, E. A.; Garbe, M.; Liu, C.; Gonzalez-de-Castro, A.; Lutz, M.; Junge, K.; Hensen, E. J. M.; Beller, M.; Lefort, L.; Pidko, E. A. Non-pincer-type manganese complexes as efficient catalysts for the hydrogenation of esters. Angew. Chem. Int Ed. 2017, 56, 7531-7534. (b) Wei, D.; Bruneau-Voisine, A.; Chauvin, T.; Dorcet, V.; Roisnel, T.; Valyaev, D. A.; Lugan, N.; Sortais, J.-B. Hydrogenation of carbonyl derivatives catalyzed by manganese complexes bearing bidentate pyridinyl-phosphine ligands. Adv. Synth. Catal. 2018, 360, 676-681.

(15) (a) Weber, S.; Stöger, B.; and Kirchner, K. Hydrogenation of nitriles and ketones catalyzed by an air-stable bisphosphine $\mathrm{Mn}(\mathrm{I})$ complex. Org. Lett. 2018, 20, 7212-7215. (b) Garduño, J. A.; García, J. J. Non-pincer $\mathrm{Mn}(\mathrm{I})$ organometallics for the selective catalytic hydrogenation of nitriles to primary amines. ACS Catal. 2019, 9, 392401.

(16) (a) Bruneau-Voisine, A.; Wang, D.; Dorcet, V.; Roisnel, T.; Darcel, C.; Sortais, J.-B. Transfer hydrogenation of carbonyl deriva- 
tives catalyzed by an inexpensive phosphine-free manganese precatalyst. Org. Lett. 2017, 19, 3656-3659. (b) Martinez-Ferraté, O.; Werlé, C.; Francio, G.; Leitner, W. Aminotriazole Mn(I) complexes as effective catalysts for transfer hydrogenation of ketones. ChemCatChem 2018, 10, 4514-4518. (c) Wang, D.; Bruneau-Voisine, A.; Sortais, J.B. Practical (asymmetric) transfer hydrogenation of ketones catalyzed by manganese with (chiral) diamine ligands. Catal. Commun. 2018, 105, 31-36.

(17) van Putten, R.; Benschop, J.; de Munck, V. J.; Weber, M.; Müller, C.; Filonenko, G. A.; Pidko, E. A. Efficient and pratical transfer hydrogenation of ketones catalyzed by a simple bidentate Mn-NHC complex. ChemCatChem 2019, 11, 5232-5235.

(18) Buhaibeh, R.; Filippov, O. A.; Bruneau-Voisine, A.; Willot, J.; Duhayon, C.; Valyaev, D. A.; Lugan, N.; Canac, Y.; Sortais, J.-B. Phosphine-NHC manganese hydrogenation catalyst exhibiting a nonclassical metal-ligand cooperative $\mathrm{H}_{2}$ activation mode. Angew. Chem. Int. Ed. 2019, 58, 6727-6731.

(19) Li, Y.; Lu, L.-Q.; Das, S.; Pisiewicz, S.; Junge, K.; Beller, M. Highly chemoselective metal-free reduction of phosphine oxides to phosphines. J. Am. Chem. Soc. 2012, 134, 18325-18329.

(20) Raba, A.; Anneser, M. R.; Jantke, D.; Cokoja, M.; Herrmann, W. A.; Kühn, F. E. Facile and scalable preparation of 2-imidazolylpyridines. Tetrahedron Lett. 2013, 54, 3384-3387.

(21) Chiu, P. L.; Lai, C.-L.; Chang, C.-F.; Hu, C.-H.; Lee, H. M. Synthesis, structural characterization, computational study, and catalytic activity of metal complexes based on tetradentate pyridine $/ N$-Heterocyclic carbene ligand. Organometallics 2005, 24, 6169-6178.

(22) For manganese NHC-pyridine-NHC complexes, see: (a) Pugh, D.; Wright, J. A.; Freeman, S.; Danopoulos, A. A. 'Pincer' dicarbene complexes of some early transition metals and uranium. Dalton Trans. 2006, 775-782. (b) Myren, T. H. T.; Lilio, A. M.; Huntzinger, C. G.; Horstman, J. W.; Stinson, T. A.; Donadt, T. B.; Moore, C.; Lama, B.; Funke, H. H.; Luca, O. R. Manganese N-heterocyclic carbene pincers for the electrocatalytic reduction of carbon dioxide. Organometallics 2019, 38, 1248-1253.

(23) For the sole example of Mn(III) complex bearing NHC core LX $_{2}$-type ligand, see: Bellemin-Laponnaz, S.; Welter, R.; Brelot, L.; Dagorne, S.; Synthesis and structure of V(V) and Mn(III) NHC complexes supported by a tridentate bis-aryloxide-N-heterocyclic carbene ligand. J. Organomet. Chem. 2009, 694, 604-606.

(24) Elangovan, S.; Garbe, M.; Jiao, H.; Spannenberg, A.; Junge, K.; Beller, M. Hydrogenation of esters to alcohols catalyzed by defined manganese pincer complexes. Angew. Chem. Int. Ed. 2016, 55, $15364-15368$.

(25) (a) Gonzalez, M. A.; Yim, M. A.; Cheng, S.; Moyes, A.; Hobbs, A. J.; Mascharak, P. K. Manganese carbonyls bearing tripodal polypyridine ligands as photoactive carbon monoxide-releasing molecules. Inorg. Chem. 2012, 51, 601-608. (b) Reddy G, U.; Axthelm, J.; Hoffmann, P.; Taye, N.; Gläser, S.; Görls, H.; Hopkins, S. L.; Plass, W.; Neugebauer, U.; Bonnet, S.; Schiller, A. Co-registered molecular logic gate with a CO-releasing molecule triggered by light and peroxide. $J$. Am. Chem. Soc. 2017, 139, 4991-4994. (c) Ganguli, K.; Shee, S.; Panja D.; Kundu, S. Cooperative Mn(I)-complex catalyzed transfer hydrogenation of ketones and imines. Dalton Trans. 2019, 48, 7358-7366.

(26) (a) Das, K.; Mondal, A.; Srimani, D. Phosphine free Mn-complex catalysed dehydrogenative $\mathrm{C}-\mathrm{C}$ and $\mathrm{C}-$ heteroatom bond formation: a sustainable approach to synthesize quinoxaline, pyrazine, benzothiazole and quinoline derivatives. Chem. Commun. 2018, 54, 10582-10585. (b) Das, K.; Mondal, A.; Srimani, D. Selective synthesis of 2-substituted and 1,2-disubstituted benzimidazoles directly from aromatic diamines and alcohols catalyzed by molecularly defined nonphosphine manganese(I) complex. J. Org. Chem. 2018, 83, 9553-9560. (c) Das, K.; Mondal, A.; Pal, D.; Srivastava, H. K.; Srimani, D. Phosphine-free well-defined $\mathrm{Mn}(\mathrm{I})$ complex-catalyzed synthesis of amine, imine, and 2,3-dihydro-1H-perimidine via hydrogen autotransfer or acceptorless dehydrogenative coupling of amine and alcohol. Organometallics 2019, 38, 1815-1825.

(27) Mede, R.; Hoffmann, P.; Klein, M.; Görls, H.; Schmitt, M.; Neugebauer, U.; Gessner, G.; Heinemann, S. H.; Popp, J.; Westerhausen, M. A water-soluble $\mathrm{Mn}(\mathrm{CO})_{3}$-based and non-toxic photoCORM for administration of carbon monoxide inside of cells. Z. Anorg. Allg. Chem. 2017, 643, 2057-2062.

(28) Passera, A.; Mezzetti, A. Mn(I) and Fe(II)/PN(H)P catalysts for the hydrogenation of ketones: a comparison by experiment and calculation. Adv. Synth. Catal. 2019, 361, 4691-4706.

(29) Although it has already been shown in related $\operatorname{Re}(\mathrm{I})$ pincer systems that $\mathrm{fac}$-isomers correspond generally to the kinetic products, the complex [17](OTf) was found to be stable in solution up to $100{ }^{\circ} \mathrm{C}$ before slowly starting to decompose, see: Wei, D.; Sadek, O.; Dorcet, V.; Roisnel, T.; Darcel, C.; Gras, E.; Clot, E.; Sortais, J.-B. Selective mono $\mathrm{N}$-methylation of anilines with methanol catalyzed by rhenium complexes: an experimental and theoretical study. J. Catal. 2018, 366, 300-309.

(30) Willot, J.; Lugan, N.; Valyaev, D. A. Binuclear $\mathrm{Cu}(\mathrm{I})$ and $\mathrm{Mn}(0)$ complexes with a tridentate pyridine-NHC-phosphane ligand in a $\mu$ $\kappa^{2} C, N-\mathrm{M} ; \kappa^{1} P$-M coordination mode. Eur. J. Inorg. Chem. 2019, 4358 4364.

(31) The only comparable value of 1.938(2) ^ was observed in $\mathrm{Mn}(\mathrm{IV})$ nitride complex beating tripodal anionic $\mathrm{PhB}(\mathrm{NHC})_{3}$ ligand, see: Ding, M.; Cutsail III, G. E.; Aravena, D.; Amoza, M.; Rouzières, M.; Dechambenoit, P.; Losovyj, Ya.; Pink, M.; Ruiz, E.; Clérac, R.; Smith, J. M. A low spin manganese(iv) nitride single molecule magnet. Chem. Sci. 2016, 7, 6132-6140.

(32) Palatinus, L.; Chapuis, G. Superflip - a computer program for the solution of crystal structures by charge flipping in arbitrary dimensions. J. Appl. Cryst. 2007, 40, 786-790.

(33) Betteridge, P. W.; Carruthers, J. R.; Cooper, R. I.; Prout, K.; Watkin, D. J. Crystals version 12: software for guided crystal structure analysis. J. Appl. Cryst. 2003, 36, 1487.

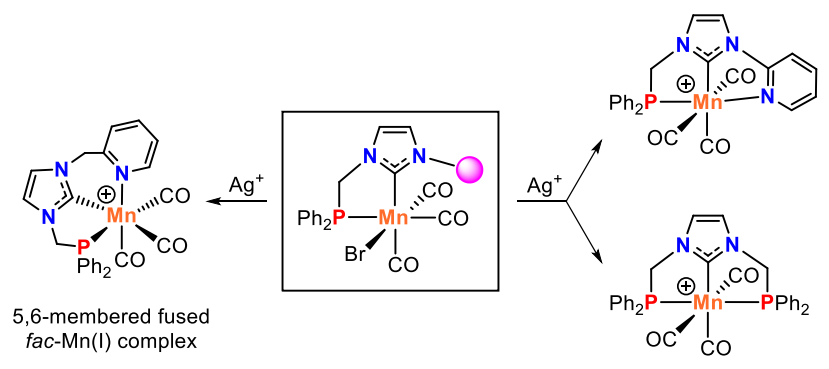

5,5-membered fused mer-Mn(I) complexes 